\title{
Mutual interference on transition of wake of circular cylinder
}

\author{
N. W. M. Ko, C. W. Law, and K. W. Lo ${ }^{\text {b) }}$ \\ Department of Mechanical Engineering, The University of Hong Kong, Hong Kong, \\ People's Republic of China
}

(Received 31 December 2003; accepted 7 May 2004; published online 7 July 2004)

\begin{abstract}
An experimental investigation was carried out in the very near wake of a circular cylinder over the Reynolds number range of $260<\operatorname{Re}<1.8 \times 10^{3}$. The aspect ratio of the cylinder was 4 . The present study concerns the regular shedding mode of the wake, in which the large-scale primary vortex sheet and the small-scale individual and pairing secondary vortices are present. Based on a new conditionally sampling technique, there is mutual interference between the high-energy small-scale secondary vortices and the development of large-scale primary vortex sheet and of the strain field of primary vortex sheet on individual secondary vortices. The pairing vortices, however, do not significantly affect the large-scale vortices. In the transition regimes within this low Reynolds number range, in which transition of two-dimensional large-scale primary vortices to three dimensionality occurs, the disturbances of the individual and pairing secondary vortices excite the separated shear layer at the most amplified mode. Coupled with mutual interference, they play an important role in the transition. Correlation with the available and additional results in the upper transition regime and in the supercritical and transcritical regimes suggests the same mechanism of transition. The mutual interference of different types of vortices in the very near wake is the key factor for transition. (C) 2004 American Institute of Physics. [DOI: 10.1063/1.1767109]
\end{abstract}

\section{INTRODUCTION}

Circular cylinder wake transition from the laminar shedding regime is sensitive to experimental conditions, the intermittent nature of the flow, and the presence of the finescale and large-scale flow structures. ${ }^{1}$ In the laminar shedding regime, besides the parallel and oblique sheddings, cellular sheddings of cells of different frequencies coexist at different spanwise locations. ${ }^{2,3}$

The transition from the two-dimensional laminar shedding regime, based on the Strouhal-Reynolds number relationship, is characterized by two distinct discontinuities. ${ }^{1,4}$ The first discontinuity at $\operatorname{Re} \approx 180$, which is hysteric, indicates the onset of the wake transition from the laminar shedding to the mode $A$ shedding. It is caused by the vortex adhesion mode and the mode $A$ instability, due to the action of elliptic vortex core instability, forming the vortex loop. The inception of vortex loop and the formation of streamwise vortex pairs, due to the deformation of primary vortices as they are shed, are at a wavelength of 3-4 diameters. ${ }^{1}$

The second discontinuity, not hysteretic, occurs at Re $\approx 230-250$, indicating intermittent swapping from the mode $A$ to mode $B$ sheddings. ${ }^{1}$ However, the regime $230<\operatorname{Re}$ $<240$ was suggested to be caused by the fading out of the mode $A$ instability. ${ }^{4}$ At $\mathrm{Re}=260$, the large-scale primary wake instability has the additional presence of fine-scale streamwise vortex structures, due to the interaction of the shear layer and wake. ${ }^{5}$ Using soap film visualization tech-

\footnotetext{
${ }^{\text {a) }}$ Present address: Environmental Protection Department, HKSAR Government.

${ }^{b)}$ Present address: Ove Arup \& Partners, L5, Festival Walk, 80 Tat Chee Avenue, Kowloon Tong, Kowloon.
}

nique, the two three-dimensional instabilities at $\operatorname{Re}=180$ and 260 are absent. $^{6}$

For the mode $A$ and mode $B$ sheddings, besides the small-scale structures, there are the large-scale threedimensional structures of vortex dislocations. ${ }^{1,5}$ These structures occur further downstream and grow to immense proportions in the streamwise and spanwise extent. They are of the order of 100 diameters.

The aspect ratio (AR) affects the two dimensionality of the wake. ${ }^{7,8}$ In the laminar shedding regime, the end effects cause the shedding of vortices, resulting in the absence or presence of the first discontinuity in the Strouhal-Reynolds number relationship. Although the recent study of Norberg ${ }^{7}$ had the lowest aspect ratio of 5, the effects of aspect ratio on laminar shedding were mainly studied at AR $>100$. Thus, the understanding on its effect in the low Reynolds number regime is still lacking.

The purpose of the present and other investigations of the present authors ${ }^{8,9}$ of low aspect ratios, $\mathrm{AR}<8$, was based on different concept of two dimensionality of cylinder flow. As the earlier concept is to maintain two dimensionality of the large-scale primary vortices in the near wake, it ignores the two dimensionality of the small-scale instability and of the secondary flow structures, if any, very near the cylinder. At higher Reynolds numbers of Re $>350$, these small-scale secondary vortices, or the Bloor-Gerrard vortices, are formed by the roll up of the separated shear layer of the cylinder. ${ }^{10-13}$ However, there was no attempt by other workers in establishing the existence of these small-scale secondary vortices in lower Reynolds number regime, $140<\operatorname{Re}$ $<260$. There was also no attempt on the mutual interaction 
between the large-scale primary and the small-scale secondary vortices and its effect on transition.

In this low Reynolds number regime, cellular sheddings of cells exist at different spanwise locations. ${ }^{2,3}$ Based on the present concept of two dimensionality of the small-scale secondary vortices, the present study aims to investigate the near wake of aspect ratio of 4 , within the range of spanwise distance of a cell.

In the upper part of the subcritical regime, $7 \times 10^{3}<\mathrm{Re}$ $<2 \times 10^{4}$, Lo and $\mathrm{Ko}^{8}$ established the upper transition between the lower and upper subcritical regime. Based on a sampling technique, the small-scale secondary vortices and their paired vortices, especially the high-energy ones, play important role in the transition. Transition involves the excitation of the separated shear layer at the most amplified mode by the disturbances associated with the secondary and paired vortices. There is mutual interference of the higherenergy small-scale vortices and the large-scale primary vortex sheet, which in turn enhances the former. These higherenergy secondary vortices enhance the chance of their pairings, which also play a dominant role in the later stage of transition. Within the supercritical and transcritical regimes, Lo and $\mathrm{Ko}^{14}$ also established the presence of secondary vortices associated with the separated shear layer in turbulent near wake. The spanwise wavelength of these high frequency secondary vortices is about 4 diameters.

In the lower transition regime, based on flow visualization laser Doppler anemometry (LDA), and digital particle image velocimetry (DPIV), Law and $\mathrm{Ko}^{9}$ confirmed the presence of small-scale secondary vortices and their pairings, even within the laminar shedding regime. At $\mathrm{AR}=4$, the two dimensionality of the separated shear layer and the newly roll-up secondary vortices is maintained. Further, switching phenomenon or bistable flow of the near wake within the lower transition regime, as those in the upper transition of the subcritical regime, occurs. The bistable flow involves the switching between the wide wake mode without the largescale primary vortex and the regular shedding mode with primary vortices. The switchings play an important role in the transition. ${ }^{9}$

The present study will further try to provide evidence on the mutual interference of the small-scale vortices with the developing large-scale vortices. Based on a new conditional sampling technique, ${ }^{8}$ the first part of the study will concern the mutual interference of these types of vortices in the regular shedding mode. The Reynolds number range was 260 $<\operatorname{Re}<1.8 \times 10^{3}$. The second part of the study will concern the correlation of the present findings with those in higher flow regimes for the establishment of mechanism of transition.

\section{EXPERIMENTAL ARRANGEMENT}

The experiments were carried out in a closed circuit water tunnel of cross section $0.43 \mathrm{~m} \times 0.43 \mathrm{~m}$. The freestream turbulence intensity was about $0.5 \%$ at the range of Reynolds number investigated. The cylinder diameter was $18 \mathrm{~mm}$. The origin of the coordinates is taken at the center of the cylinder. The $x$ axis coincides with the direction of the flow, the $z$ axis with the cylinder axis, and the $y$ direction normal to these two. Rectangular end plates of $x / d=2.6$ upstream, $x_{t} / d$ $=7.0$ downstream, width of $6.0 \mathrm{~d}$, and sharp leading edge were used. ${ }^{9,15,16}$ The aspect ratio of the cylinder was 4 . At this aspect ratio the Kelvin-Helmholtz instability is two dimensional in the early stage of transition, resulting in the formation of the small-scale Bloor-Gerrard secondary vortices. ${ }^{9}$ A compromise on the wall effects was adopted, as a reasonably thick shear layer was desirable for velocity measurements. The present aspect ratio, blockage ratio, and local turbulence intensity were within the ranges used by past researchers. ${ }^{17,18}$

The Reynolds number range, based on the diameter and freestream velocity $U_{0}$, was $260<\operatorname{Re}<1.8 \times 10^{3}$, covering the transitions from the regime of periodic laminar wake to different subregimes of the present aspect ratio.

Hot film anemometer was used to measure the streamwise mean velocity in the water tunnel. DANTEC 55R32 hot film probe and DANTEC 56C16 constant temperature anemometer were used. The regime of study was at $0.8 \leqslant x / d$ $\leqslant 2.5$, mainly within the formation length of $1.5 d-5 d$ of the large-scale primary vortices. ${ }^{7,11,12,19,20}$ Due to the small size of the separated shear layer, acquisition of the signal was only made at the traverse position at which its amplitude was the highest. The signal was acquired through a Data Translation 2821-G-16SE digital data acquisition board via a PC 80486. The sampling rate was $25 \mathrm{~Hz}$. The sampling time varied and was in the range of $10^{4} \mathrm{~s}$. Since the frequency ratios of the secondary and primary vortices, as will be shown later, are less than 4 , the sampling rate is acceptable. Data processing was carried out in a PC Pentium II. The boundary layer near the separation point of the cylinder was measured by a DANTEC $55 \times$ modular LDA and FVA 58 N40 with a Coherent $6 \mathrm{~W}$ Argon-ion laser.

For LDA measurements, streamwise component of the velocity close to the cylinder was obtained. Measurements were made at $x / d=0$ and $0.5 \leqslant y / d \leqslant 1.06$ and at least 500 samples were obtained at every sample point. The fiber-optic probe was moved with a computer-controlled traverse table, such that the measurement point covered the region with a $0.1 \mathrm{~mm}$ or $0.0056 d$ resolution.

Flow visualization, based on the introduction of dyes of different color, was carried out in a water tunnel. If the dyes were introduced at $\theta=15^{\circ}$ from the front stagnation point, the flow structures in the near wake were visualized. The pictures were captured by a Nikon $801 \mathrm{~S}$ camera with a frame speed of 3.3 frames per second. Continuous images were also acquired by a charged couple device (CCD) camera and a Panasonic video recorder. A Data Translation 2869 video decoder/encoder, Data Translation 2871 frame grabber, and Data Translation 2878 image processing cards were used to transfer the video signals to a PC $80486 \mathrm{DX}$. For the present low Reynolds number range, the streaklines nearly indicate the vortical structures in the near wake of the cylinder. ${ }^{19,21}$

Simultaneous measurements of hot film signals and video flow visualization pictures were also made at the Reynolds number of 320 . The hot film was located at $x / d=1.2$, $y / d=-0.7, z / d=0$. The purpose was to validate the velocity 
measurements from the hot film being associated with the vortices.

A video based digital particle image velocimetry (DPIV) was used to obtain the instantaneous velocity field in the near wake. The present method, based on that of Willert and Gharib, ${ }^{22}$ computed directly the spatial cross correlation of the two images to obtain the velocity data. A $2 \mathrm{~mm}$ thick pulse laser sheet was generated by a $6 \mathrm{~W}$ Argon-ion laser to illuminate the seed particles of silicon powder of $20 \mu \mathrm{m}$ diameter. The image was sharpened by a precision beam chopper before being captured by the CCD camera and the video recorder in $25 \mathrm{~Hz}$. Based on the $u$ and $v$ velocities in the $x$ and $y$ directions, the vorticity $\omega_{z}$ in the $z$ direction is

$$
\omega_{\mathrm{z}}=\left(\frac{\partial v}{\partial x}-\frac{\partial u}{\partial y}\right) .
$$

The Stokes theorem was used to carry out the noise suppression. $^{23}$

For the present system, the spatial resolution was 6.4 pixels $/ \mathrm{mm}$. Keane and Adrian ${ }^{24}$ used different experimental variables and Monte Carlo simulation in estimating the velocity uncertainty. Their results were, thus, based on the broad criteria for the optimized system performance for single exposure images and the cross correlation DPIV analysis. ${ }^{24}$ Employing similar methods, the present estimated maximum overall velocity uncertainty was about $6 \%$. The maximum overall vorticity uncertainty, based on the velocity field reconstruction, was about $16 \%$.

Additional measurements at $4 \times 10^{3}<\operatorname{Re}<4 \times 10^{4}$ in the upper transition regime were made in a low speed wind tunnel of test section $0.4 \mathrm{~m} \times 0.4 \mathrm{~m}$. The freestream turbulence intensity was about $0.2 \%$ at the freestream mean velocity of $20 \mathrm{~m} / \mathrm{s} .{ }^{8}$ The cylinder diameter was $50 \mathrm{~mm}$. Two rectangular end plates with leading edge of $2.5 d$ and trailing edge of $4.5 d$ were located at $2 d$ from the tunnel wall. ${ }^{12}$ As the shear layer was very thin, compromise on the wall effects was also made. The aspect ratio of the cylinder was 4 . The configurations were also within the ranges used by past researchers. ${ }^{17,18}$

The size of the separated shear layer very near the cylinder of the present study was small, of the order of $0.1 d$. It rendered more uncertainty in adopting other more elaborated measurement methods, which required bigger sensors, such as those of two velocity components or of vorticity. Thus, with least interference, single hot wire with its one component of velocity was adopted for the following conditional sampling measurements. However, other methods, as described above, were carried out to substantiate the use of single hot wire. As the regime of the present study was 0.8 $\leqslant x / d \leqslant 2.5$, which is within the formation regions of not only the large-scale primary vortices, but also of the smallscale secondary vortices, two dimensionality might still basically be found. Thus, the use of streamwise velocity was believed to be acceptable.

The single hot-wire measurements were made with a wire diameter of $5 \mu \mathrm{m}$ and length of $2 \mathrm{~mm}$. Due to the small thickness of the separated shear layer in the formation region, a three-wire probe of wire lateral spacing of $1 \mathrm{~mm}$ was

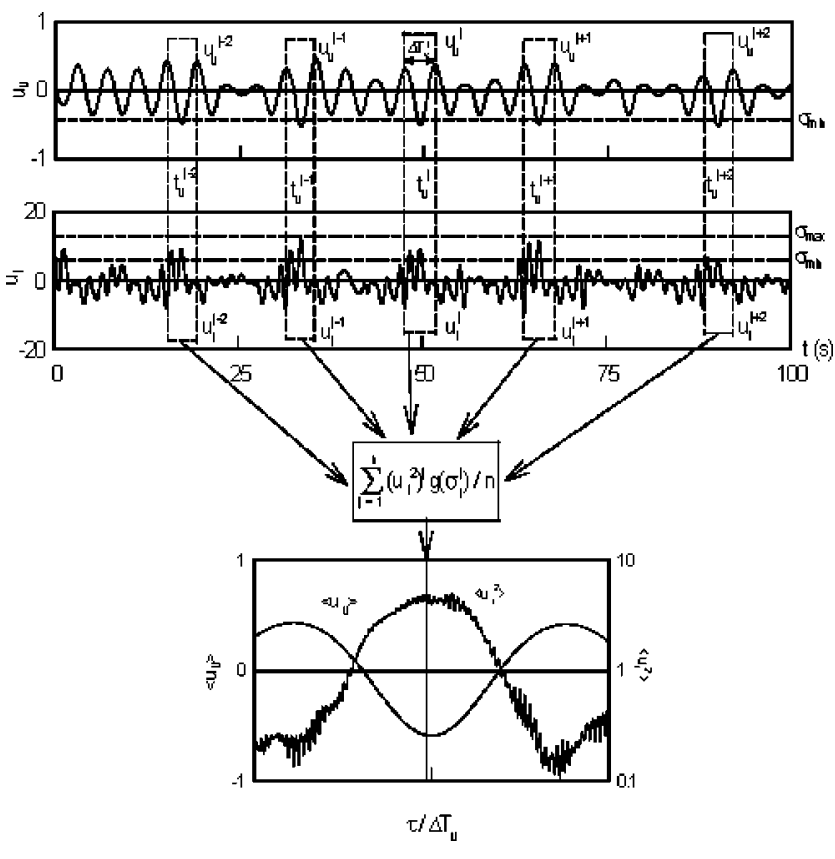

FIG. 1. Definitions of recovered time histories.

used. ${ }^{8}$ Among the three signals obtained in the shear layer, only the highest amplitude one was selected. The hot-wire probe was mounted on a computer controlled table for positioning in the $x, y, z$ directions. The signal from the constant temperature anemometer was acquired at a rate of 5000 samples per second through a Data Translation 2821 digital data acquisition board. A sampling time was of $500 \mathrm{~s}$. The data were then transferred to the Workstation DEC5000/200 PXG and Pentium II Processor for further analysis.

A new conditional sampling scheme was developed to reveal the mutual interference of the secondary vortices at the frequency $f_{\mathrm{i}}$ and the primary vortex sheet at $f_{\mathrm{v}} \cdot{ }^{8}$ In this scheme, the trough of the velocity $u_{\mathrm{v}}$ of the primary vortex sheet time history was located first (Fig. 1). In order to determine the effect of the strength of the secondary vortices on the primary vortex sheet, the velocity signal of the secondary vortices in the trough region was sampled according to a threshold level. Two threshold levels, $1<\sigma_{\mathrm{i}}<2$ and $\sigma_{\mathrm{i}}>2$ were adopted for the $260<\operatorname{Re}<1.8 \times 10^{3}$ and three threshold levels, $1<\sigma_{\mathrm{i}}<2,2<\sigma_{\mathrm{i}}<3$, and $\sigma_{\mathrm{i}}>3$ were used for the 4 $\times 10^{3}<\operatorname{Re}<4 \times 10^{4} . \sigma_{\mathrm{i}}$ is the threshold level ratio of the instantaneous velocity $u_{\mathrm{i}}$ to the rms value $u_{\mathrm{i}}^{\prime}$ of the secondary vortices. Based on the threshold level of the secondary vortices, the primary vortex sheet was also sampled. Since the formation and presence of the secondary vortices on the primary vortex sheet were not stationary, some cancellation might occur during averaging. Instead of sampling the streamwise velocity $u_{\mathrm{i}}$ of the secondary vortices, their streamwise energy $u_{\mathrm{i}}^{2}$ was adopted.

The triple decomposition of the fluctuating velocity field yields, ${ }^{25}$

$$
u=u_{\mathrm{v}}+u_{\mathrm{i}}+u_{\mathrm{r}},
$$

where subscripts $v, i$, and $r$ denote the primary vortex sheet, secondary vortex, and random fluctuating components, re- 
spectively. As the shedding frequencies of the primary and secondary vortices are different, those of the former $f_{\mathrm{v}}$ and of the latter $f_{\mathrm{i}}$ can be identified by spectral analysis. Thus, in the lower Reynolds number regime, as their frequencies are closer, 1/8 octave band-pass filtering at their corresponding spectral peak frequencies was performed. For the higher Reynolds number regime, 1/3 octave band-pass filtering was carried out.

At the minimum velocity $\left(u_{\mathrm{v}}\right)_{\min }$, the time of the $j$ th sample of the primary vortex sheet is $t_{\mathrm{v}}^{\mathrm{j}}$ (Fig. 1). The secondary vortices within the time period of $\left(t_{\mathrm{v}}^{\mathrm{j}}-\Delta T_{\mathrm{v}}^{\mathrm{j}} / 2\right)<t$ $<\left(t_{\mathrm{v}}^{\mathrm{j}}+\Delta T_{\mathrm{v}}^{\mathrm{j}} / 2\right)$ were scanned for their maxima in the determination of the threshold level $\sigma_{\mathrm{i}}^{\mathrm{j}} . \Delta T_{\mathrm{v}}^{\mathrm{j}}$ is the period of the successive maximum velocities of the primary vortex sheet. As there are different threshold levels, the function

$$
\begin{array}{ll}
g\left(\sigma_{\mathrm{i}}^{\mathrm{j}}\right)=1 & \text { if } \sigma_{\min }<\sigma_{\mathrm{i}}<\sigma_{\max }, \\
g\left(\sigma_{\mathrm{i}}^{\mathrm{j}}\right)=0 & \text { if } \sigma_{\text {min }}>\sigma_{\mathrm{i}} \text { or } \sigma_{\mathrm{i}}>\sigma_{\text {max }} .
\end{array}
$$

Thus, the valid samples of the secondary vortices within the time period of $\left(t_{\mathrm{v}}^{\mathrm{j}}-\Delta T_{\mathrm{v}}^{\mathrm{j}} / 2\right)<t<\left(t_{\mathrm{v}}^{\mathrm{j}}+\Delta T_{\mathrm{v}}^{\mathrm{j}} / 2\right)$ are acquired. The recovered streamwise energy of $n$ samples of primary vortex sheet, based on the alignment with $t_{\mathrm{v}}^{\mathrm{j}}$, is

$$
\left\langle u_{\mathrm{i}}^{2}\right\rangle=\sum_{\mathrm{j}=\mathrm{n}}\left(u_{\mathrm{i}}^{\mathrm{j}}\right)^{2} g\left(\sigma_{\mathrm{i}}^{\mathrm{j}}\right) / n .
$$

The recovered streamwise velocity of the primary vortex sheet, based on the alignment with $t_{\mathrm{v}}^{\mathrm{j}}$, is

$$
\left\langle u_{\mathrm{v}}\right\rangle=\sum_{\mathrm{j}=\mathrm{n}} u_{\mathrm{v}}^{\mathrm{j}} g\left(\sigma_{\mathrm{i}}^{\mathrm{j}}\right) / n .
$$

The total number of ensembles of each threshold level was greater than 500 .

In the recovery of the paired secondary vortices, the same conditional sampling scheme was adopted, except that the threshold levels were based on the $\sigma_{\mathrm{i} / 2}$ at the subharmonic frequency $f_{\mathrm{i}} / 2 .{ }^{9}$ The recovered streamwise energy of paired secondary vortices of $n$ samples of primary vortex sheet, based on the alignment with $t_{\mathrm{v}}^{\mathrm{j}}$, is

$$
\left\langle u_{\mathrm{i} / 2}^{2}\right\rangle=\sum_{\mathrm{j}-\mathrm{n}}\left(u_{\mathrm{i} / 2}^{\mathrm{j}}\right)^{2} g\left(\sigma_{\mathrm{i} / 2}^{\mathrm{j}}\right) / n .
$$

The total number of ensembles of each threshold level was greater than 200 .

\section{RESULTS AND DISCUSSION}

Based on the definitions of the large-scale primary vortex associated with the global instabilities ${ }^{10,19}$ and of the small-scale Bloor-Gerrard secondary vortex associated with the separated shear layer, ${ }^{12}$ the roll up of the KelvinHelmholtz instabilities occurs at $1<x / d<3$ for $\mathrm{Re}=300$ and at $0.7<x / d<2.2$ for $\operatorname{Re}=1.3 \times 10^{3} .{ }^{9}$ The corresponding formation length of the primary vortices are at $1.5<x / d<4.7$ for $\operatorname{Re}=300$ and at $1.8<x / d<3.8$ for $\operatorname{Re}=1.3 \times 10^{3}$.

The earlier study established the stabilizing effect of aspect ratio on transition. ${ }^{9}$ In the lower transition regime, at $\mathrm{AR}=4$, the laminar vortex shedding regime occurs at $\mathrm{Re}$ $<320$, the mode $A$ shedding subregime at $320<\operatorname{Re}<600$,

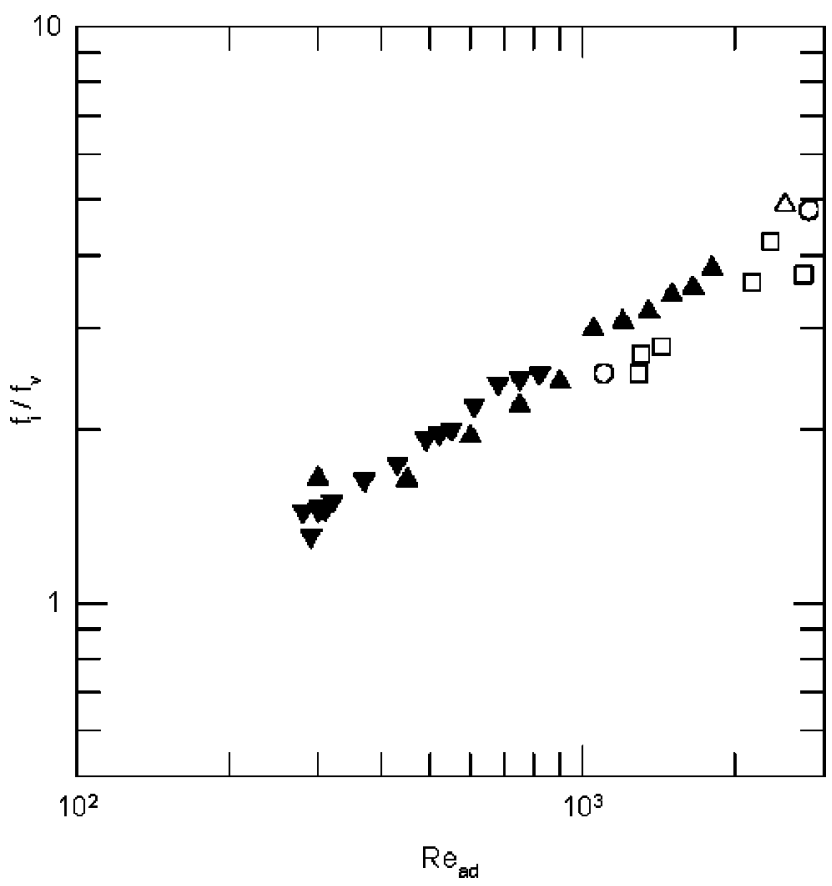

FIG. 2. Secondary vortex and primary vortex frequency ratios. $\bigcirc$, Bloor (1964) (Ref. 10), $\square$, Wei and Smith (1986) (Ref. 13); $\triangle$, Kourta et al. (1987) (Ref. 12), $\mathbf{\Lambda}, \mathrm{AR}=4.0$, present study; $\mathbf{\nabla}, \mathrm{AR}=4.0$, present study, hot film measurement.

and the mode $B$ shedding subregime at $\operatorname{Re}>600$. The critical Reynolds number $\operatorname{Re}_{\mathrm{cr}}=320$ is higher than the $\mathrm{Re}_{\mathrm{cr}}=180$ at AR $>200 ., 26,27$

The frequency ratios $f_{\mathrm{i}} / f_{\mathrm{v}}$ of primary and secondary vortices with the adjusted Reynolds number $\mathrm{Re}_{\mathrm{ad}}$ are shown in Fig. 2. The $\operatorname{Re}_{\mathrm{ad}}=\operatorname{Re} \times\left(\operatorname{Re}_{\mathrm{cr}}\right)_{\mathrm{AR}} /\left(\mathrm{Re}_{\mathrm{cr}}\right)_{\mathrm{AR}>9}$, allowing for the difference in the $\operatorname{Re}_{\mathrm{cr}}$ at $\mathrm{AR}<9$ from that at $\mathrm{AR}>9$. The $\left(\mathrm{Re}_{\mathrm{cr}}\right)_{\mathrm{AR}>9}$ is assumed to be $180 .{ }^{1}$ The present results within the lower transition regime of $\mathrm{AR}=4$ are of the same trend as those of higher Reynolds numbers of others workers. ${ }^{1,10,12,13}$ In the regular shedding mode, within the lower transition regime, the frequency of the secondary vortices is only slightly higher than that of the primary vortices. This may be the cause for the wrong identification of these small-scale secondary vortices and their absence by other workers. The above phenomena indicate that within the lower transition, in the regular shedding mode of the wake, there are small-scale secondary vortices within the formation region of the large-scale primary vortices. With their presence in the formation region of the primary vortices, especially with the same direction of vorticity, one would expect a certain amount of mutual interference. Based on conditional sampling technique, the following sections will try to establish the mutual interference of secondary and Strouhal vortices and their effect on the transitions to different regimes.

\section{A. Conditional sampling results}

Simultaneous streamwise filtered velocities at $f_{\mathrm{v}}$ of the primary and $f_{\mathrm{i}}$ of the secondary vortices at $x / d=1.2, y / d$ $=-0.7, z / d=0$, as obtained by the hot film and the sketches from the video flow visualization at three time instants are 

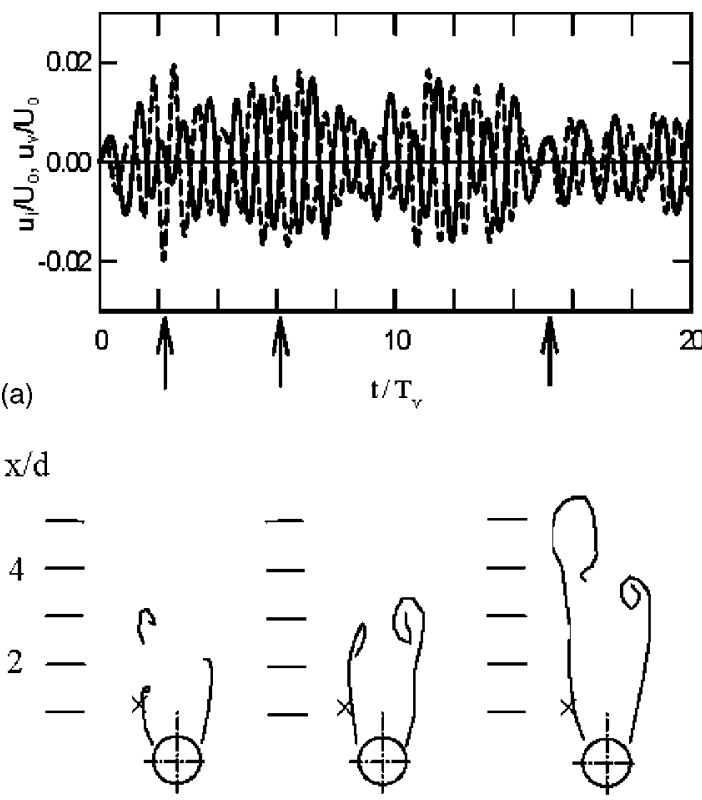
(b) $\mathrm{t} / \mathrm{T}_{\mathrm{v}}=2.2$
(c) $\mathrm{t} / \mathrm{T}_{\mathrm{v}}=6.1$
(d) $\mathrm{t} / \mathrm{T}_{\mathrm{v}}=15.1$

FIG. 3. Simultaneous time histories of primary vortex sheet and secondary vortices and sketches of flow visualization. Hot film at $x / d=1.2, y / d$ $=-0.7, z / d=0 ;-$, primary vortex sheet; ------, initial secondary vortex.

shown in Figs. 3(a)-3(d). It tries to illustrate the streamwise velocity measurements in the shear layer within the very near wake and the detection of the passages of secondary and Strouhal vortices. The two $f_{\mathrm{i}}$ velocity peaks earlier than the first instant $\left(t / T_{\mathrm{v}}=2.2\right)$ are associated with the two secondary vortices at $x / d \approx 1.5$ and 3.3 [Figs. 3(a) and 3(b)]. These two secondary vortices do not indicate any induction towards the center of the cylinder. At the second time instant $\left(t / T_{\mathrm{v}}\right.$ $=6.1$ ), the primary vortex at $x / d \approx 2.7$ is associated with the earlier $f_{\mathrm{v}}$ velocity peak [Figs. 3(a) and 3(c)]. The primary vortex is induced towards the center. At the third time instant $\left(t / T_{\mathrm{v}}=15.1\right)$, both velocity peaks are of lower amplitudes [Figs. 3(a) and 3(d)]. The flow visualization does not show any vortex being formed. The simultaneous results, therefore, validate that the filtered streamwise velocities are associated with the passages of vortices.

At $\operatorname{Re}<320$, in the regime of the beginning of transition of laminar vortex shedding to wake transition regime, the streamwise velocity time trace of the secondary vortices at $f_{\mathrm{i}}$ seems to be irregular [Fig. 4(a)]. Besides the low frequency modulation, both velocity time traces show occasional deviation of the period of successive velocity peaks from those of more regular peaks, suggesting the variation in their frequencies. Based on the conditional sampling technique, at Re $=290$, within the laminar vortex shedding regime, there is a rapid increase in the number of low-energy vortices (Fig. 5). The distribution of the recovered energies $\left\langle u_{\mathrm{i}}^{2}\right\rangle / u_{\mathrm{i}}^{2}$ of the secondary vortices are not periodic [Fig. 6(a)].

At $\mathrm{Re}_{\mathrm{cr}}=320$, the lower transition of laminar vortex shedding to wake transition regime, low frequency modulation is found in both traces and the more dominant peaks of both traces are about anti phased [Fig. 4(b)]. At this Reynolds number, there are concurrent appearances of dominant
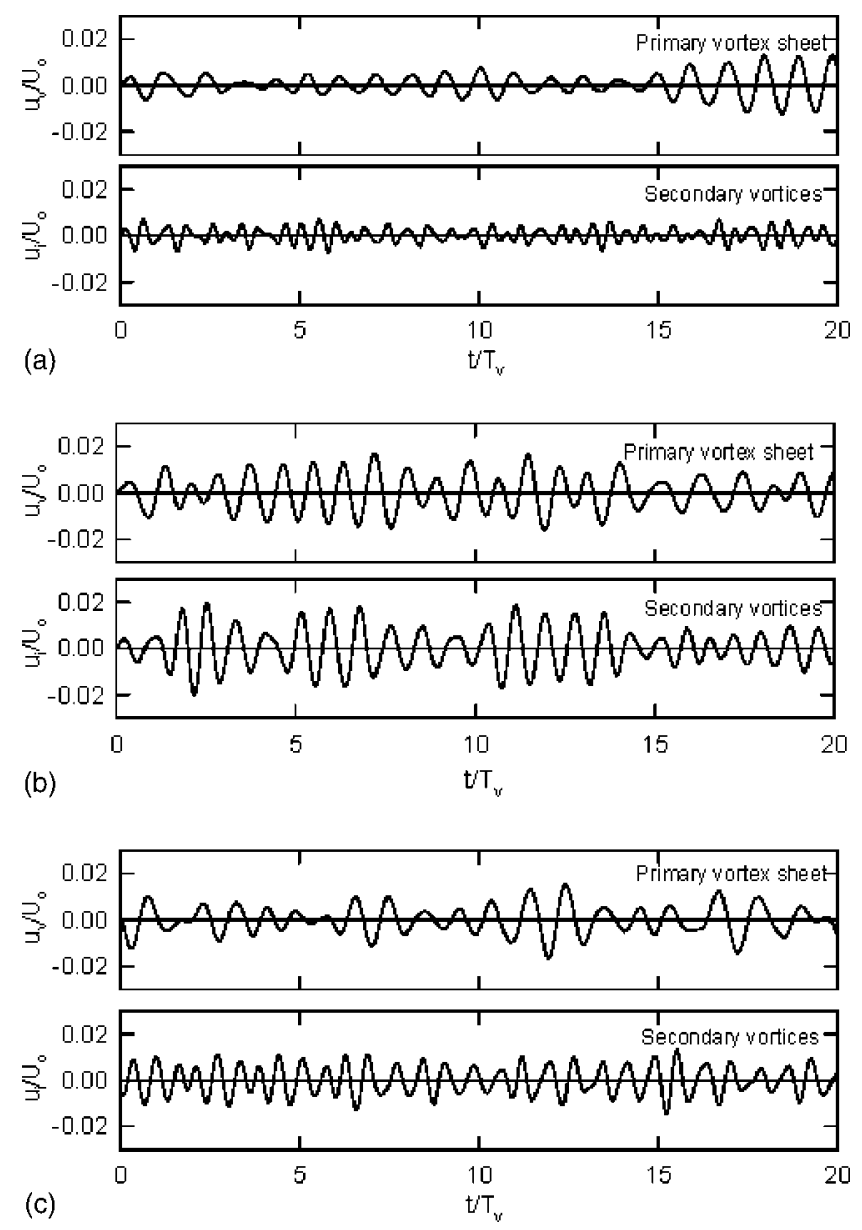

FIG. 4. Time histories of bandpassed filtered streamwise velocities of primary vortex sheet and secondary vortices in regular shedding mode at $x / d$ $=1.2, y / d=0.7, \mathrm{AR}=4.0$. (a) $\mathrm{Re}=290$; (b) $\operatorname{Re}=320$; (c) $\mathrm{Re}=680$.

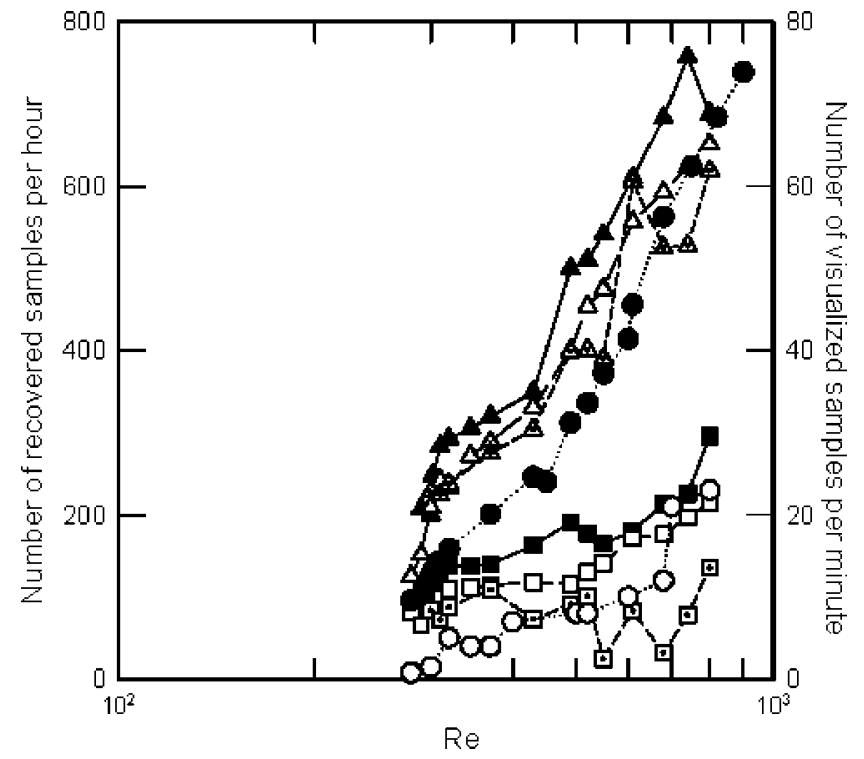

FIG. 5. Number of secondary vortices and paired secondary vortices recovered and visualized. $\boldsymbol{\Delta}, x / d=1.2,1<\sigma_{\mathrm{i}}<2 ; \mathbf{\square}, x / d=1.2,2<\sigma_{\mathrm{i}}<10 ; \triangle$, $x / d=2.5, \quad 1<\sigma_{\mathrm{i} / 2}<2 ; \square, x / d=2.5,2<\sigma_{\mathrm{i} / 2}<10 ; \quad \Delta, x / d=3.0,1<\sigma_{\mathrm{i} / 2}$ $<2 ; \square, x / d=3.0,2<\sigma_{\mathrm{i} / 2}<10 ; \bullet$, single secondary vortex (dye visualization results); $\bigcirc$, paired secondary vortex (dye visualization results). 

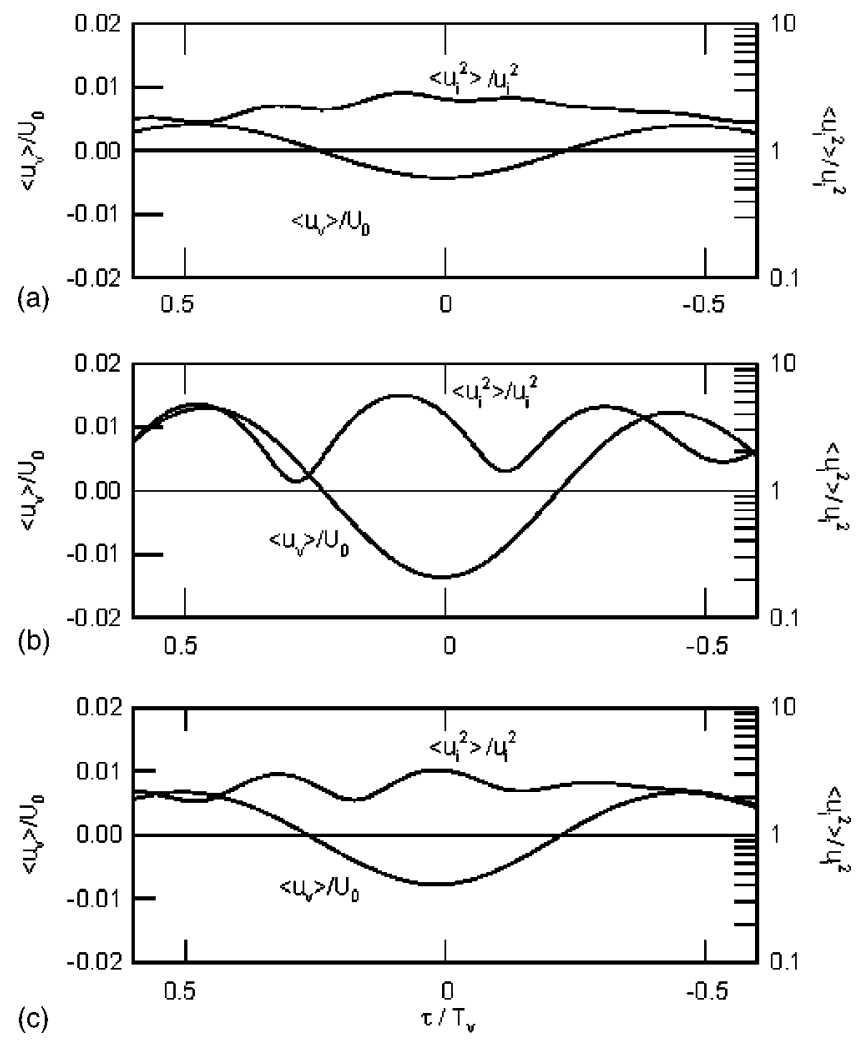

FIG. 6. Recovered time histories of streamwise velocity of primary vortex sheet and secondary vortex energies at $2<\sigma_{\mathrm{i}}<10$ and $x / d=1.2$. (a) $\operatorname{Re}$ $=290$; (b) $\operatorname{Re}=320$; (c) $\operatorname{Re}=680$.

peaks of both traces at $5<t / T_{\mathrm{v}}<8$ and $11<t / T_{\mathrm{v}}<14$, before the modulation. There is also a rapid increase in the highenergy secondary vortices (Fig. 5). The recovered streamwise velocity peaks associated with the secondary vortices at $x / d=1.2$ have significantly more low-energy ones $\left(1<\sigma_{\mathrm{i}}\right.$ $<2)$ than high-energy ones $\left(2<\sigma_{\mathrm{i}}<10\right)$. Moreover, the distribution of the secondary vortices is periodic [Fig. 6(b)].

At $420<\operatorname{Re}<720$, the transition between mode $A$ and mode $B$ shedding subregimes comprises finer-scale streamwise vortices and large-scale three-dimensional distortion. ${ }^{1}$ There is a rapid increase in the number of the low-energy vortices (Fig. 5). The drops or smaller increases in the number of secondary vortices at $\operatorname{Re} \approx 500$ and 700 mark the transitions of subregimes. There is also variation in the phase difference between the peaks of the secondary vortices and those of the primary sheet. The phase difference varies from nearly in phased of the upstream vortex to about antiphased of the downstream one. The difference in the periods of these two recovered time traces indicates the higher frequency of the small-scale vortices. At $\operatorname{Re}=680$, just within the mode $B$ subregime, the secondary vortex time trace has less low frequency modulation, while there is significant variation of the primary vortices [Fig. 4(c)]. As the number of secondary vortices is significantly higher than that of primary vortices, their antiphase at the critical Reynolds number no longer exists. The secondary vortices become less periodic and have a peak in the $\left\langle u_{\mathrm{i}}\right\rangle$ [Fig. 6(c)].

At $\operatorname{Re}=800$, within the mode $B$ subregime, the rate of increase of the low-energy secondary vortices is higher than

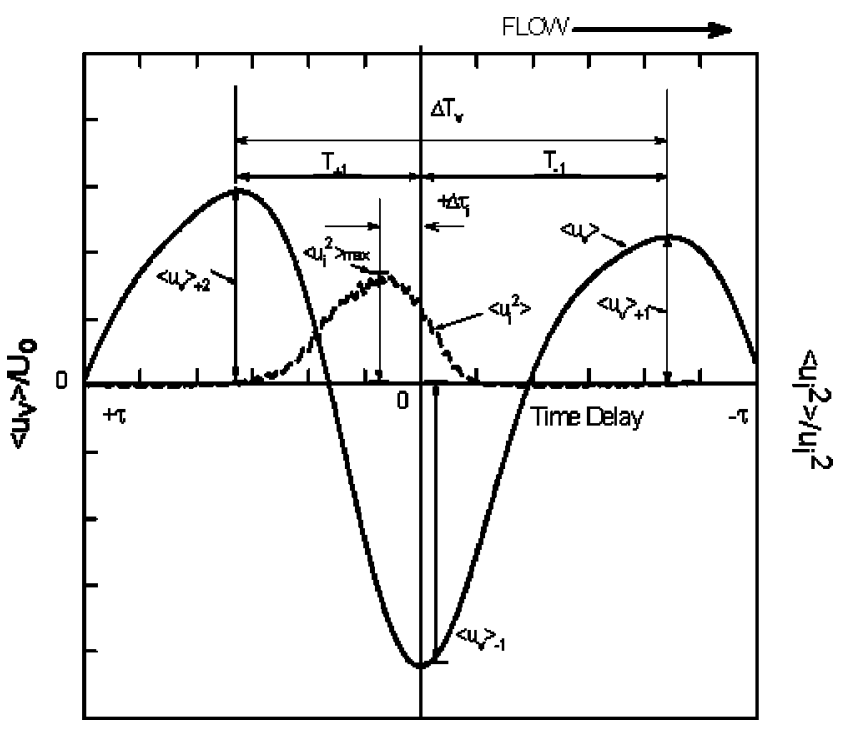

FIG. 7. Definitions of recovered primary vortex sheet velocity and secondary vortex energies; —_, primary vortex sheet velocity; -----, secondary vortex energy.

that of the high-energy ones, resulting in significantly higher number of low-energy secondary vortices at this streamwise position (Fig. 5). Their ratio increases from about 2 at $\mathrm{Re}$ $=280$ to 3.5 at $\operatorname{Re}=750$.

As the rapid increases of the secondary vortices occur in the earlier part of the transition, the phenomenon implies the significance of the rapid increase in these small-scale secondary vortices and their role in the transition.

\section{B. Mutual interference of secondary vortices and Strouhal vortex sheet}

Based on the definitions shown in Fig. 7, the following sections will try to establish the mutual interference of the secondary vortices and the primary vortex sheet. The variations of the recovered maximum energy $\left\langle u_{\mathrm{i}}^{2}\right\rangle_{\max } / u_{\mathrm{i}}^{2}$ of the secondary vortices at $x / d=1.2$ for $1<\sigma_{\mathrm{i}}<2$ and $2<\sigma_{\mathrm{i}}$ $<10$ are shown in Fig. 8. The lower transition involves a rapid increase to a peak in the $\left\langle u_{\mathrm{i}}^{2}\right\rangle_{\max } / u_{\mathrm{i}}^{2}$ of the high-energy secondary vortices $\left(2<\sigma_{\mathrm{i}}<10\right)$ at $300<\operatorname{Re}<310$. The increase of the low-energy secondary vortices $\left(1<\sigma_{\mathrm{i}}<2\right)$ is not obvious. The transition between the mode $A$ and mode $B$ subregimes at $\mathrm{Re} \approx 600$ also has a peak, though the rate is more moderate.

The above peaks also appear as troughs in the variations of the recovered primary vortex sheet amplitude ratio $\left\langle u_{\mathrm{v}}\right\rangle_{+1} /\left\langle u_{\mathrm{v}}\right\rangle_{+2}$, as recovered by the high-energy secondary vortices $\left(2<\sigma_{\mathrm{i}}<10\right)$ (Fig. 9). ${ }^{9}$ Those recovered by the lowenergy secondary vortices $\left(1<\sigma_{\mathrm{i}}<2\right)$ do not indicate similar phenomenon. It, thus, suggests a certain amount of mutual interference between the high-energy secondary vortices with the primary vortex sheet such that the streamwise velocity of the latter is affected. Figure 10 shows at $\left\langle u_{\mathrm{i}}^{2}\right\rangle_{\max } / u_{\mathrm{i}}^{2}>1$, it is the high-energy secondary vortices affecting the development of the primary vortex sheet. The present results and those of $\mathrm{Lo}$ and $\mathrm{Ko}^{8}$ in the upper transi- 


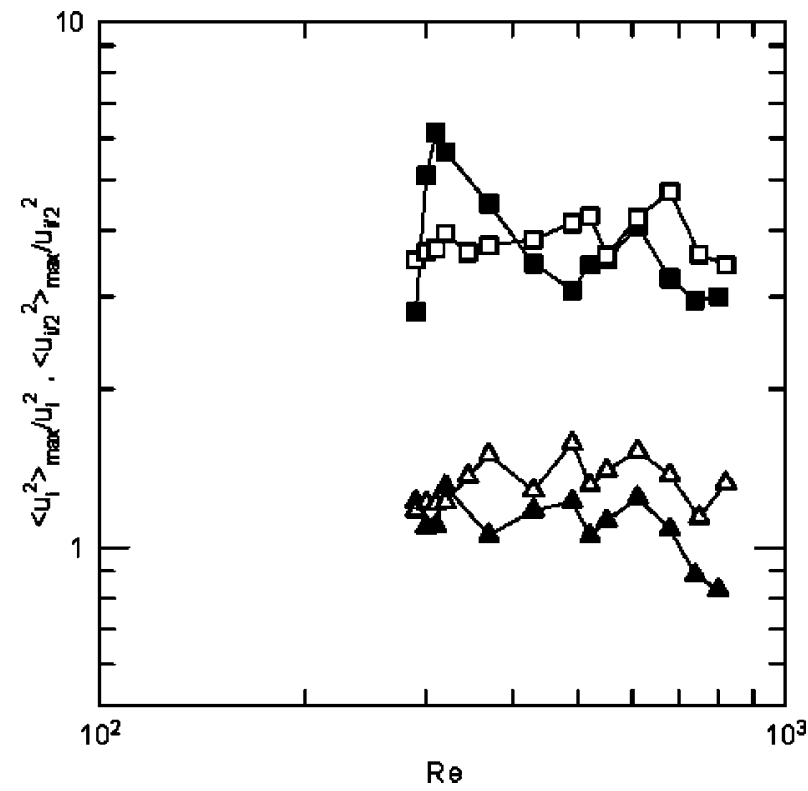

FIG. 8. Maximum recovered energies of secondary and paired secondary vortices at different threshold levels. $\boldsymbol{\Delta}, 1<\sigma_{\mathrm{i}}<2 ; \mathbf{\square}, 2<\sigma_{\mathrm{i}}<10 ; \triangle, 1$ $<\sigma_{\mathrm{i} / 2}<2 ; \square, 2<\sigma_{\mathrm{i} / 2}<10$.

tion regime are also included in the figure. They are of the same trend as those of lower transition.

It is interesting to find the agreement of different sets of results in these two regimes of lower and upper transitions. The agreement indicates the same effect of mutual interference between the energy of the secondary vortices and the streamwise velocity development of the primary vortex sheet, irrespective of the regime. The trend further suggests that at $\left\langle u_{\mathrm{i}}^{2}\right\rangle_{\max } / u_{\mathrm{i}}^{2}<1$, the $\left\langle u_{\mathrm{v}}\right\rangle_{+1} /\left\langle u_{\mathrm{v}}\right\rangle_{+2} \approx 1$. At $\left\langle u_{\mathrm{i}}^{2}\right\rangle_{\max } / u_{\mathrm{i}}^{2}$ $>1$, the $\left\langle u_{\mathrm{v}}\right\rangle_{+1} /\left\langle u_{\mathrm{v}}\right\rangle_{+2}<1$, implying either the downstream

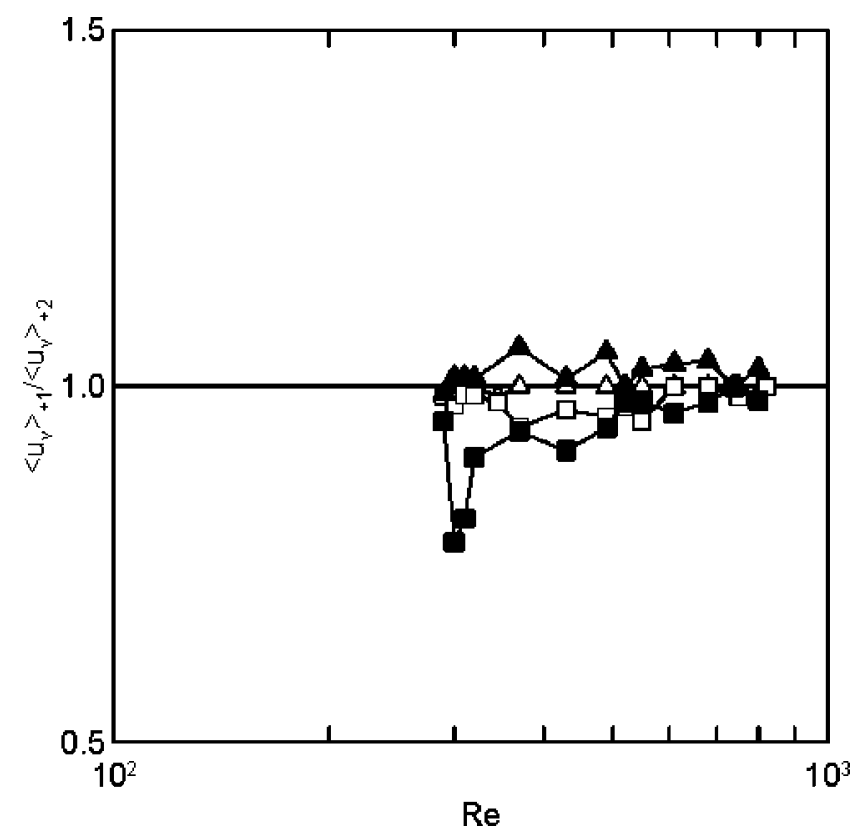

FIG. 9. Recovered amplitude ratios of primary vortex sheet by secondary vortices at $x / d=1.2$ and of primary vortices by paired secondary vortices at $x / d=2.5 . \boldsymbol{\Lambda}, 1<\sigma_{\mathrm{i}}<2 ; \mathbf{\square}, 2<\sigma_{\mathrm{i}}<10 ; \triangle, 1<\sigma_{\mathrm{i} / 2}<2 ; \square, 2<\sigma_{\mathrm{i} / 2}<10$.

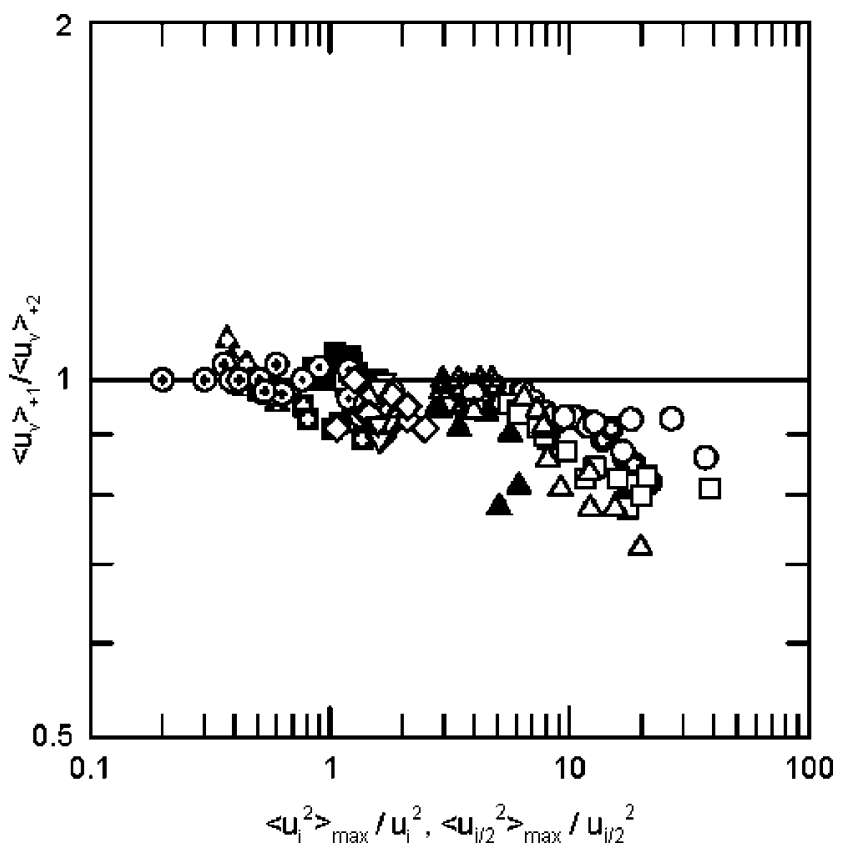

FIG. 10. Recovered amplitude ratios of primary vortex sheet with recovered peak energy of secondary and paired vortices. $\boldsymbol{\Lambda}, 1<\sigma_{\mathrm{i}}<2, \operatorname{Re}<10^{3} ; \boldsymbol{\square}$, $2<\sigma_{\mathrm{i}}<10, \operatorname{Re}<10^{3} ; \boldsymbol{A}, 1<\sigma_{\mathrm{i}}<2,10^{3}<\operatorname{Re}<10^{5} ; \boldsymbol{\#}, 2<\sigma_{\mathrm{i}}<3,10^{3}<\operatorname{Re}$ $<10^{5} ; \boldsymbol{\theta}, 3<\sigma_{\mathrm{i}}, 10^{3}<\operatorname{Re}<10^{5} ; \mathbb{A}, 1<\sigma_{\mathrm{i} / 2}<2, \operatorname{Re}<10^{3} ; \boxplus, 2<\sigma_{\mathrm{i} / 2}$ $<10, \operatorname{Re}<10^{3}$; Lo and Ko (2001) (Ref. 8) $1<\sigma_{\mathrm{i}}<2,10^{3}<\operatorname{Re}<10^{5}: \oplus$, Group I; $2<\sigma_{\mathrm{i}}<3,10^{3}<\operatorname{Re}<10^{5}: \odot$, Group I; $\diamond$, Group II; $\nabla$, Group III; $3<\sigma_{\mathrm{i}}, 10^{3}<\operatorname{Re}<10^{5}: 0$, Group I; $\square$, Group II; $\triangle$, Group III.

amplitude $\left\langle u_{\mathrm{v}}\right\rangle_{+1}$ is smaller or the upstream amplitude $\left\langle u_{\mathrm{v}}\right\rangle_{+2}$ is greater, due to the presence of the high-energy small-scale vortices. As the recovered upstream velocity ratio $\left[\left\langle u_{\mathrm{v}}\right\rangle_{+2}\right]_{2<\sigma_{i}<10} /\left[\left\langle u_{\mathrm{v}}\right\rangle_{+2}\right]_{1<\sigma_{i}<2}$ is greater than unity (not shown here), it indicates that the high-energy secondary vortices amplify the upstream vortex sheet amplitude, while the downstream one is not affected. This phenomenon is similar to the findings of acoustically excited separated shear layer of a smooth cylinder at the initial secondary vortex frequency ${ }^{28}$ The primary vortex is amplified and the formation length is reduced, due to the corresponding increase in the fluid entrainment into the primary vortex sheet. Thus, in the transition regimes, this enhanced interference effect of the more intense high-energy secondary vortices may partly be responsible for the change in the peak amplitude and the low frequency modulation of the streamwise velocity of the primary vortex sheet.

The development and presence of the secondary vortices in the trough of the primary vortex sheet are associated with the evolution of the vorticity concentration. These secondary vortices are associated with the vortex layer between the large-scale transverse vortex structures being unstable to the strain field induced by the transverse vortices. ${ }^{29,30}$ For twodimensional vortex sheet near the separation point and of near constant convection velocity, the rate of linear strain in the streamwise direction $\partial u / \partial x$ is proportional to $\partial u / \partial t .^{31}$ Thus, the ratios of the upstream maximum absolute linear strain rates due to the high-and low-energy secondary vortices at $\quad x / d=1.2, \quad\left|d\left\langle u_{\mathrm{v}}\right\rangle_{+2} / d t\right|_{\max , 2<\sigma_{i}<10} /$ $\left|d\left\langle u_{\mathrm{v}}\right\rangle_{+2} / d t\right|_{\max , 1<\sigma_{i}<2}$, indicate its effect on the developing 


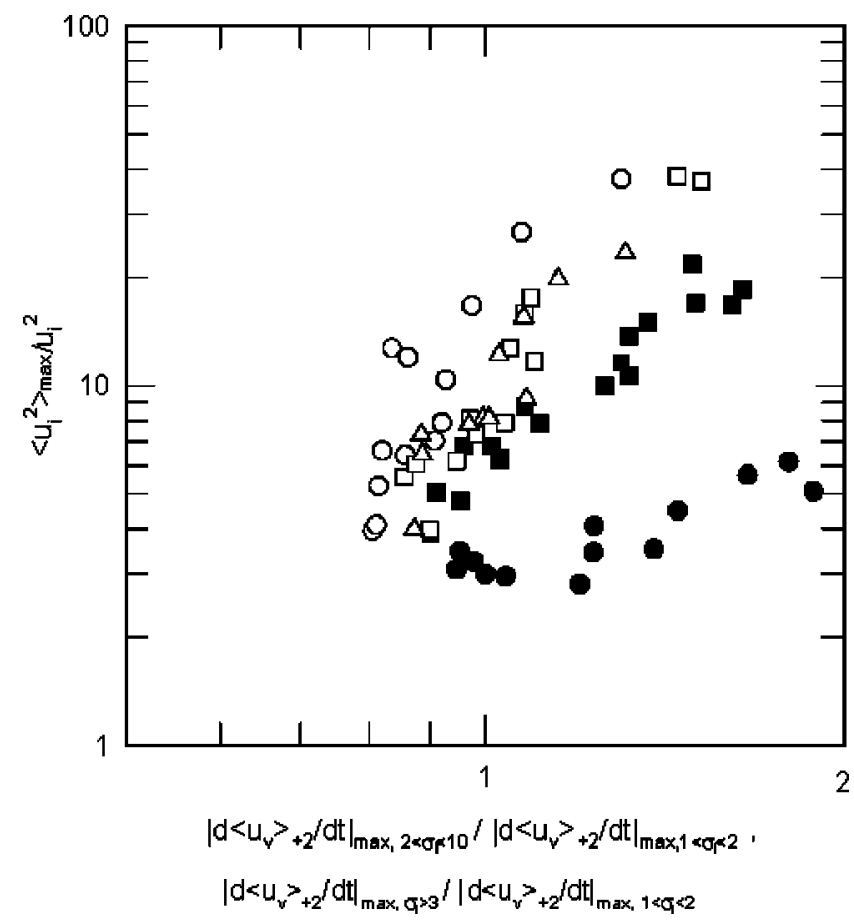

FIG. 11. Recovered peak energies of secondary vortices with linear strain rate ratios of primary vortex sheet. $\bullet$, $\left|d\left\langle u_{\mathrm{v}}\right\rangle_{+2} / d t\right|_{\max , 2<\sigma i<10} /\left|d\left\langle u_{\mathrm{v}}\right\rangle_{+2} / d t\right|_{\max , 1<\sigma i<2}, \quad x / d=1.2, \quad 250<\operatorname{Re}$ $<10^{3} ; \mathbf{\square}, \quad\left|d\left\langle u_{\mathrm{v}}\right\rangle_{+2} / d t\right|_{\max , 3<\sigma i} /\left|d\left\langle u_{\mathrm{v}}\right\rangle_{+2} / d t\right|_{\max , 1<\sigma i<2}, \quad x / d=0.3, \quad 4$ $\times 10^{3}<\operatorname{Re}<4 \times 10^{4}$; Lo and Ko (2001) (Ref. 8): upstream peak: $3<\sigma_{\mathrm{i}}$, $10^{3}<\operatorname{Re}<10^{5}$ : $\bigcirc$, Group I; $\square$, Group II; $\triangle$, Group III.

secondary vortices (Fig. 11). The results in the upper transition regime, from the lower subcritical to the upper subcritical regime, of Lo and $\mathrm{Ko}^{8}$ are also shown in the figure. They are higher than those of the same regime of the present study. It may be because the streamwise position of the present study was at $x / d=0.3$, while that of the earlier study was at $x / d=0.2$. In this higher Reynolds number regime, the formation length of the secondary vortices is small and the streamwise position is more critical. The streamwise position of $x / d=0.2$ is at the position just after the formation of secondary vortices. Thus, the slightly further downstream position means that the effect of the strain is on the developed secondary vortices, rather than on the developing vortices. In addition, at this position there may be the beginning of pairings and of the decay of the small-scale vortices. Nevertheless, it is still interesting to find that the higher strain field of the more developed primary vortex sheet results in more intense evolution of the secondary vortices. As the energy of the secondary vortices is generally higher, one would then expect the effect is significantly higher in the upper transition regime than in the lower regime.

The distribution of the phase shift, $\Delta \tau_{\mathrm{i}} / \Delta T_{\mathrm{v}}$, between the peak of the recovered maximum secondary vortex energy and the trough of primary vortex sheet at $2<\sigma_{\mathrm{i}}<10$ shows peaks of about 0.1 at $\operatorname{Re} \approx 300$ and 0.05 at $\operatorname{Re} \approx 600$ (not shown here). At $\operatorname{Re} \approx 400$ and 640 there is no phase shift, implying that after the transitions the newly formed secondary vortices are located at the trough of the primary vortex sheet. The above peak Reynolds numbers agree with those of

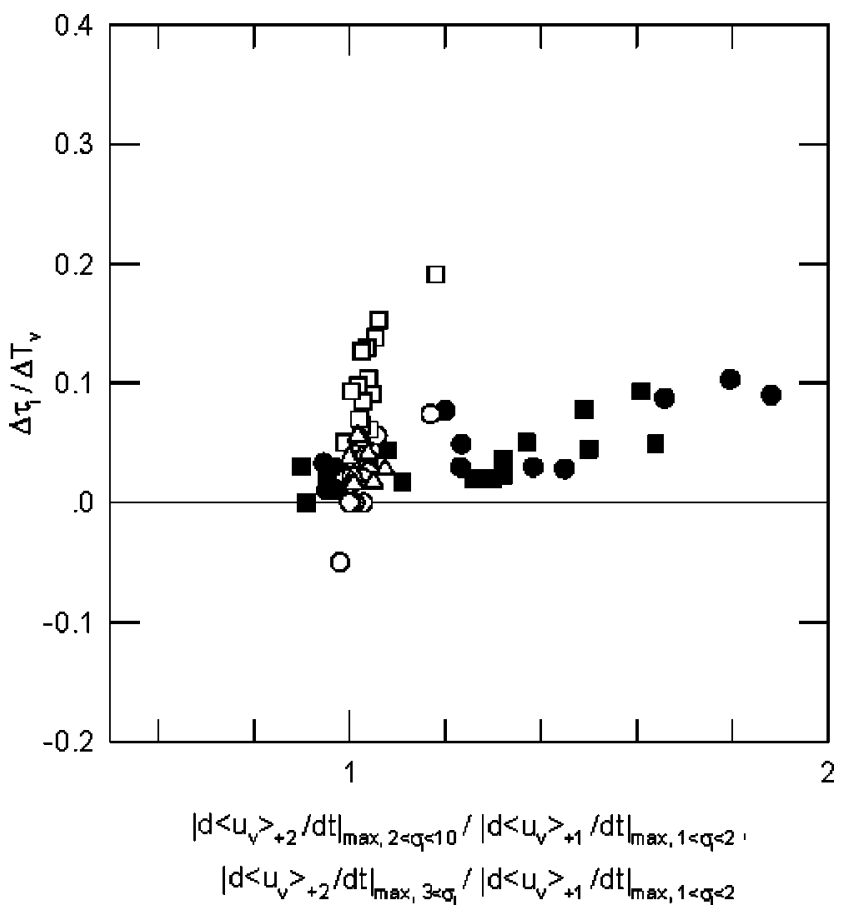

FIG. 12. Phase shifts with linear strain rate ratios of primary vortex sheet. -, $\left|d\left\langle u_{\mathrm{v}}\right\rangle_{+2} / d t\right|_{\max , 2<\sigma i<10} /\left|d\left\langle u_{\mathrm{v}}\right\rangle_{+1} / d t\right|_{\max , 1<\sigma i<2}, \quad x / d=1.2, \quad 250<\operatorname{Re}$ $<10^{3} ; \quad \mathbf{\square}, \quad\left|d\left\langle u_{\mathrm{v}}\right\rangle_{+2} / d t\right|_{\max , 3<\sigma i} /\left|d\left\langle u_{\mathrm{v}}\right\rangle_{+1} / d t\right|_{\max , 1<\sigma i<2}, \quad x / d=0.3, \quad 4$ $\times 10^{3}<\operatorname{Re}<4 \times 10^{4}$; Lo and Ko (2001) (Ref. 8): upstream peak: $3<\sigma_{\mathrm{i}}$, $10^{3}<\operatorname{Re}<10^{5}: \bigcirc$, Group I; $\square$, Group II; $\triangle$, Group III.

the energy $\left\langle u_{\mathrm{i}}^{2}\right\rangle_{\max } / u_{\mathrm{i}}^{2}$ (Fig. 8), suggesting that during transition the newly roll-up secondary vortices are affected by the primary vortex sheet on which they are located.

The variation of phase shift, $\Delta \tau_{\mathrm{i}} / \Delta T_{\mathrm{v}}$, with the ratio of upstream and downstream maximum linear strain rates $\left|d\left\langle u_{\mathrm{v}}\right\rangle_{+2} / d t\right|_{\max , 2<\sigma_{i}<10} /\left|d\left\langle u_{\mathrm{v}}\right\rangle_{+1} / d t\right|_{\max , 1<\sigma_{i}<2}$ of the primary vortex sheet is shown in Fig. 12. The variation of the present study and that of the upstream peak of Lo and $\mathrm{Ko}^{8}$ in the upper transition regime are included. The strain not only affects the energy of the secondary vortices (Fig. 11), but also the location of these small-scale vortices on the primary vortex sheet. The effect is higher in the upper transition regime than in the lower one. This implies that under the effect of the strain field of the primary vortex sheet, the secondary vortices are further upstream, enhancing the interaction effect of the secondary vortices.

The above has shown the high-energy secondary vortices affect the development of the large-scale vortex sheet, before the latter rolls up into primary vortices. Without the effect of secondary vortices, one would expect that $\left\langle u_{\mathrm{v}}\right\rangle_{+1} /\left\langle u_{\mathrm{v}}\right\rangle_{+2}$ is greater than unity, as the downstream waveform amplitude would be higher due to its development. Some of the amplitude ratios are slightly greater than unity at $\left\langle u_{\mathrm{i}}^{2}\right\rangle_{\max } / u_{\mathrm{i}}^{2}<1$ (Fig. 10), implying that some of the low-energy secondary vortices do not significantly inhibit the large-scale vortex sheet development. The ratio of less than unity at $\left\langle u_{\mathrm{i}}^{2}\right\rangle_{\max } / u_{\mathrm{i}}^{2}>1$ indicates that the high-energy secondary vortices amplify the upstream $\left\langle u_{\mathrm{v}}\right\rangle_{+2}$ of the primary vortex sheet.

These secondary vortices also affect the strain rate of the 


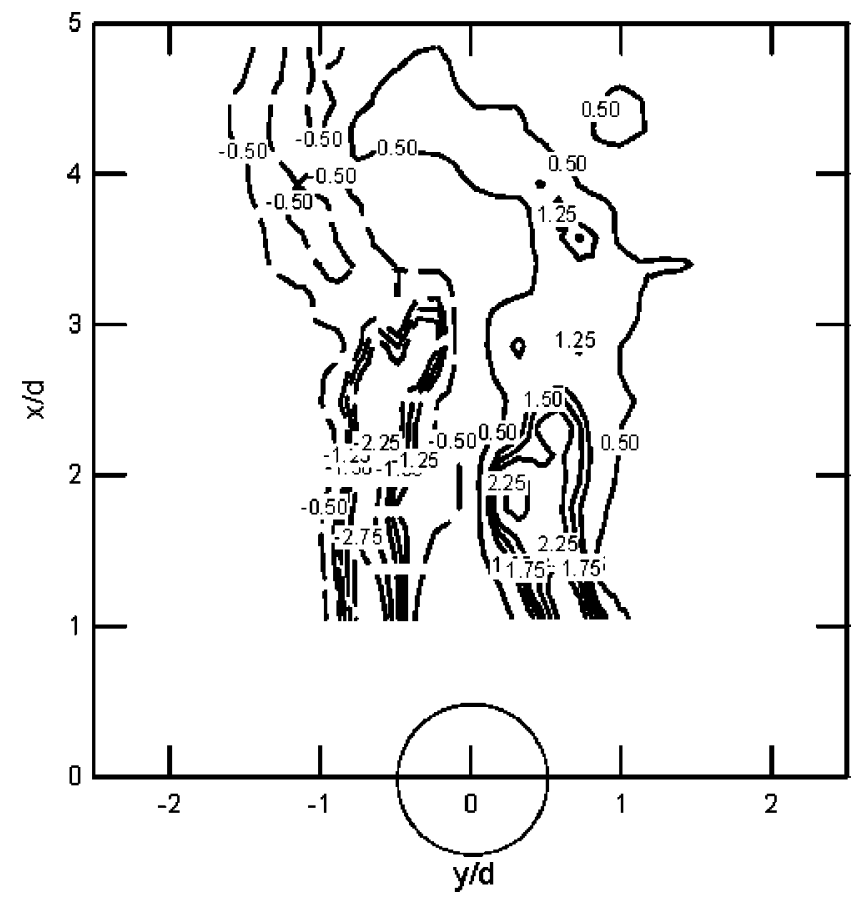

FIG. 13. Normalized vorticity contour plot of regular vortex shedding mode at $\mathrm{AR}=4.0, \mathrm{Re}=320$. Maximum overall vorticity uncertainty $=16 \%$.

primary vortex. The higher the energy, the higher is the effect on the strain rate. Further, the higher strain rate induces higher energy of these small-scale vortices. The present observation of the effect of strain field also supports the findings of the effect of weak low frequency forcing on the formation of higher frequency instability. ${ }^{32,33}$ The present findings of the transition regimes are also similar to the more rapid secondary vortex evolution and deformation, due to the presence of a heavy forcing effect of the primary vortices on the region of initial disturbance growth. ${ }^{20}$

\section{Mutual interference of pairing vortices and Strouhal vortex sheet}

The normalized vorticity $\omega_{\mathrm{z}} /\left(U_{\mathrm{o}} / d\right)$ contours, as estimated by the DPIV method, show two secondary vortices that undergo pairing process on both sides of the wake (Fig. 13). The spectrum of the streamwise velocity indicates the spectral peak at the frequency $f_{\mathrm{i}} / 2$ of half of the peak frequency $f_{\mathrm{i}}$ of the secondary vortices (not shown here). By defining the secondary vortex pairing location as the streamwise distance between the center of the cylinder and the location where the trailing secondary vortex catches up with the leading secondary vortex, ${ }^{34}$ the regime of pairings depends on Reynolds number. It varies from $2<x / d<4.5$ at $\mathrm{Re}=300$ to $1.2<x / d<2.3$ at $\mathrm{Re}=1.8 \times 10^{3}$. It is further downstream than that of the secondary vortices.

The recovered numbers of pairings of the two threshold levels at $x / d=2.5$ and 3 are shown in Fig. 5. From flow visualization, the average numbers of pairings per minute at $2<x / d<4$ are also shown. The period of the count was 60 min. The pairings recovered at the low threshold level 1 $<\sigma_{\mathrm{i} / 2}<2$ are more numerous than those at $2<\sigma_{\mathrm{i} / 2}<10$. Their ratio is about 1.3 at $\operatorname{Re}=280$ to about 2.4 at $\mathrm{Re}$
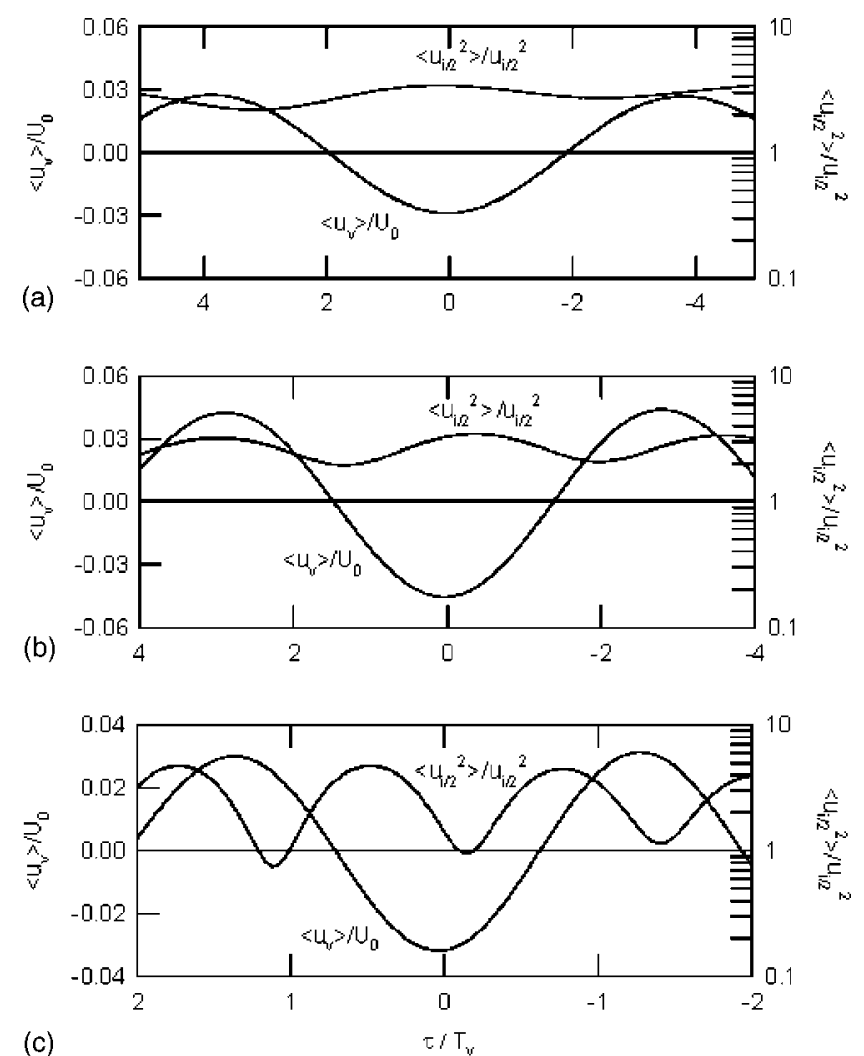

FIG. 14. Time histories of bandpassed filtered streamwise velocities of primary vortices and paired secondary vortices in regular shedding mode at $2<\sigma_{\mathrm{i} / 2}<10, x / d=2.5, y / d=0.7, \mathrm{AR}=4.0$. (a) $\operatorname{Re}=290$; (b) $\mathrm{Re}=320$; (c) $\mathrm{Re}=680$.

$=750$. These ratios are slightly lower than those of individual secondary vortices of 2 and 3.5. This means that there are lesser intense pairing vortices. However, for those at 1 $<\sigma_{\mathrm{i} / 2}<2$, the rapid increases in the number of pairings in the lower transition regime, $280<\operatorname{Re}<320$, and in the transition regime between mode $A$ and mode $B, 450<\operatorname{Re}<700$, are of the same trend as those of flow visualization. Further, they are of similar trend as those of individual secondary vortices.

The recovered maximum energy ratios $\left\langle u_{\mathrm{i} / 2}^{2}\right\rangle / u_{\mathrm{i} / 2}^{2}$ at $x / d$ $=2.5$ of the pairing vortices at the two threshold levels are shown in Fig. 8. The low-energy pairing vortices $\left(1<\sigma_{\mathrm{i} / 2}\right.$ $<2)$ seem to have higher energy ratio than that of the individual secondary vortices. It is expected, as the streamwise energy of paired vortices is higher than that of single vortices. ${ }^{35,36}$ During pairing, the fluid entrainment and circulation are enhanced. ${ }^{29,37}$

For the high-energy pairing vortices $\left(2<\sigma_{\mathrm{i} / 2}<10\right)$, however, the maximum energy ratio seems to be lower than that of individual secondary vortices at $\operatorname{Re}<400$. At higher Reynolds numbers, it is the reverse. This lower energy ratio may partly be due to the streamwise position of $x / d=2.5$, being at the earlier regime of pairings, in which the pairing process has not been completed. ${ }^{9}$

The recovered time histories of the streamwise velocities of the primary vortex sheet and pairing secondary vortices at $x / d=2.5$ in the three subregimes are shown in Fig. 14. At 


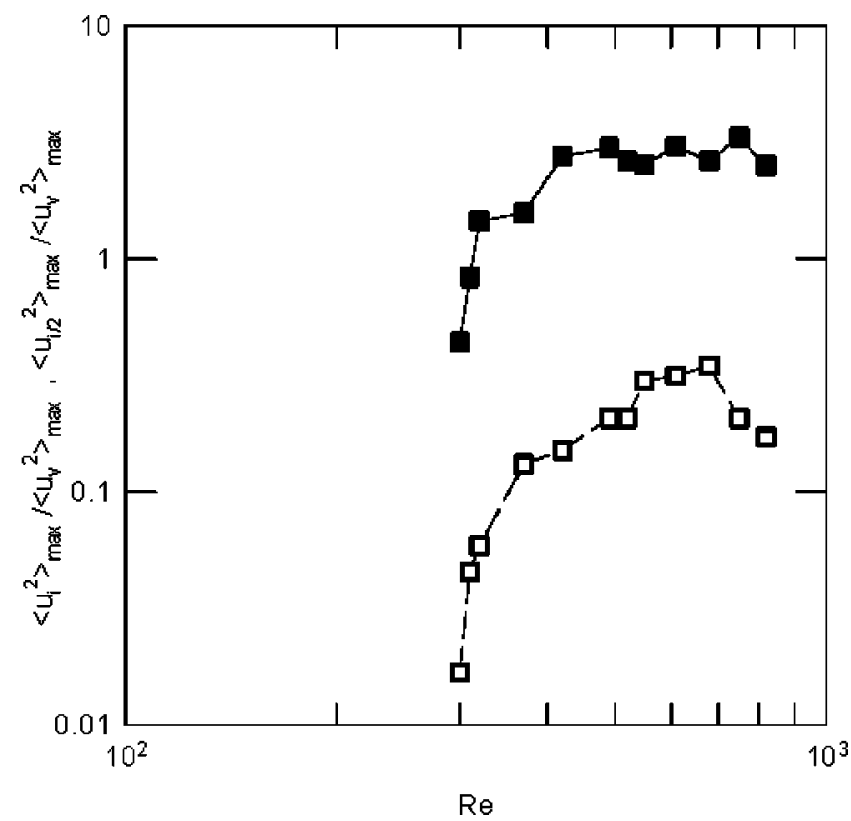

FIG. 15. Recovered peak energy ratios with Reynolds number. $\mathbf{\square}$, secondary vortices at $x / d=1.2 ; \square$, paired secondary vortices at $x / d=2.5$.

$\mathrm{Re}=290$, the distribution of the pairing vortices are not periodic [Fig. 14(a)]. At the $\mathrm{Re}_{\mathrm{cr}}=320$, it is more periodic [Fig. 14(b)]. At $\mathrm{Re}=680$, periodicity is found [Fig. 14(c)].

The effect of $\left\langle u_{\mathrm{i} / 2}^{2}\right\rangle_{\max } / u_{\mathrm{i} / 2}^{2}$ of the pairing vortices on $\left\langle u_{\mathrm{v}}\right\rangle_{+1} /\left\langle u_{\mathrm{v}}\right\rangle_{+2}$ is shown in Fig. 10. At the highest value of $\left\langle u_{\mathrm{i} / 2}^{2}\right\rangle_{\max } / u_{\mathrm{i} / 2}^{2} \approx 4$, the high-energy pairing vortices seem not to affect the primary vortex sheet. Further, the recovered maximum energy $\left\langle u_{\mathrm{i} / 2}^{2}\right\rangle_{\max }$ of the pairing vortices at this streamwise position is an order of magnitude lower than the energy $\left\langle u_{\mathrm{i}}^{2}\right\rangle_{\max }$ of the individual secondary vortices (Fig. 15). The weaker pairing vortices in the earlier pairing regime do not affect significantly the development of the primary vortices.

The distribution of the phase shift $\Delta \tau_{\mathrm{i} / 2} / \Delta T_{\mathrm{v}}$ of the high-energy pairing vortices with the trough of primary vortex sheet is inside the negative domain (not shown here). The pairing vortices have their energy peak slightly downstream of the streamwise mean velocity trough of primary vortices. This streamwise position of $x / d=2.5$ is near the end of the formation region of primary vortices and the vortex sheet has newly rolled up into vortices. Near this position vortex pairings start to occur. These pairing vortices have not completed the process.

The above results illustrate the mutual interference of the high-energy individual secondary vortices and the primary vortex sheet in the regular shedding mode of the wake. The low-energy individual secondary vortices and the pairing secondary vortices, even the high-energy ones, do not play significant role. However, the above findings do not indicate the cause for the more rapid development of the individual secondary vortices in the lower transition regime and in the transition regime between the mode $A$ and mode $B$ subregimes. $\mathrm{x} / \mathrm{d}$

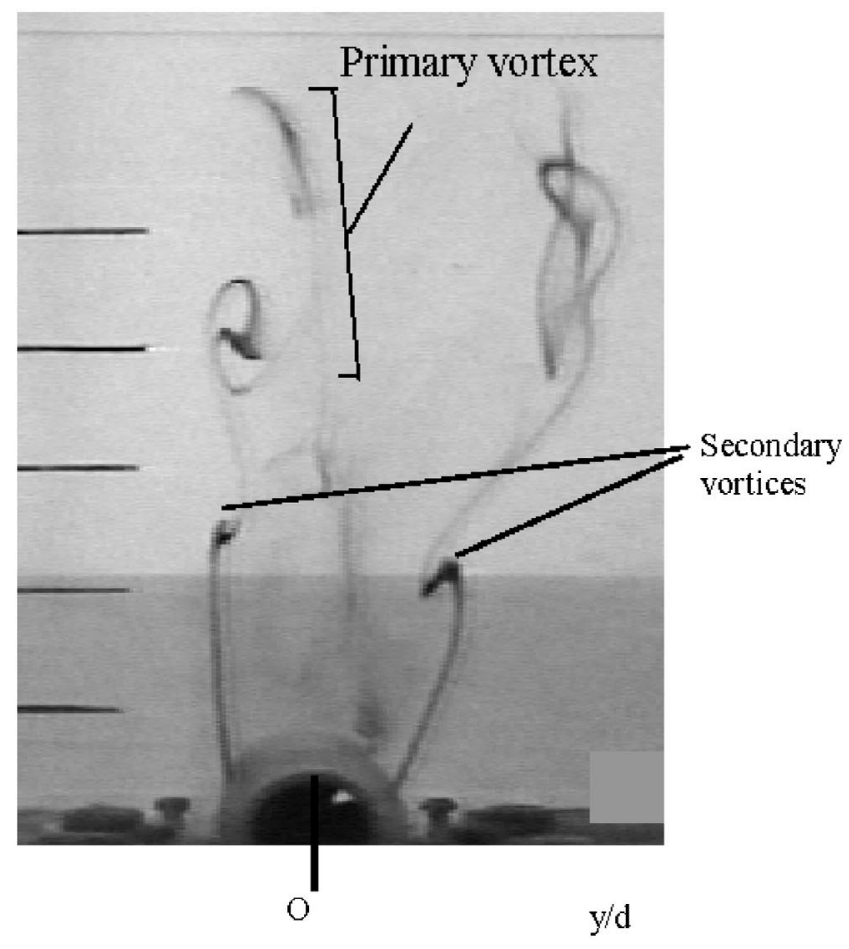

(a)

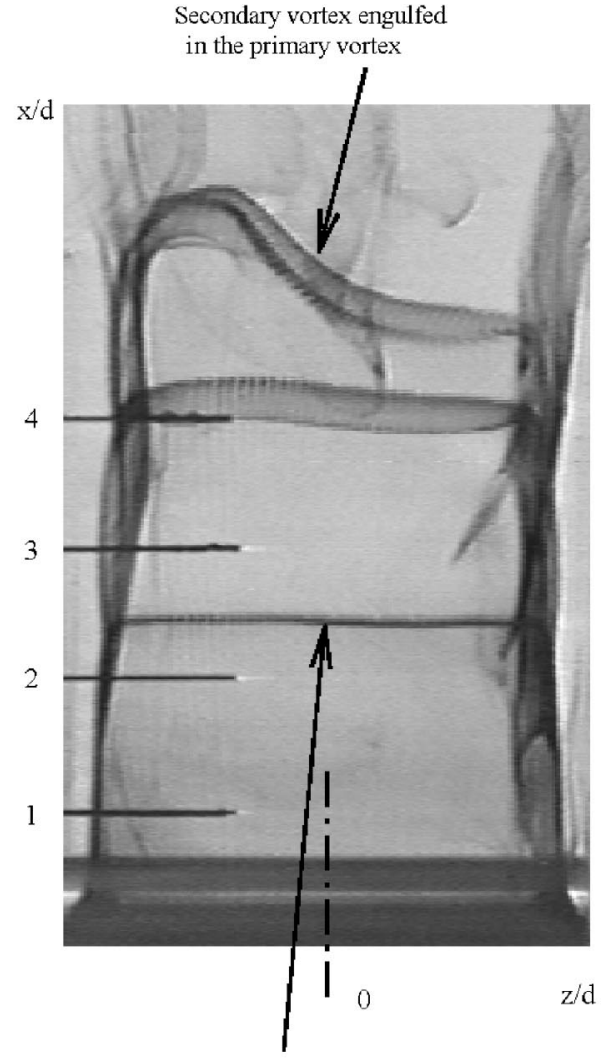

(b)

Secondary vortex

FIG. 16. Flow visualization at $\mathrm{AR}=4.0, \mathrm{Re}=300$. (a) Streamwise; (b) spanwise. 


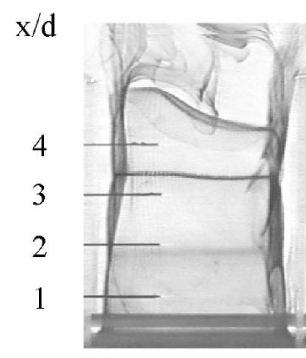

(a)

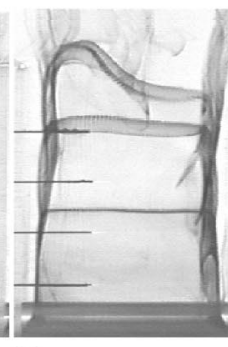

(b)

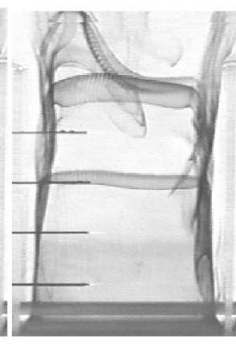

(c)

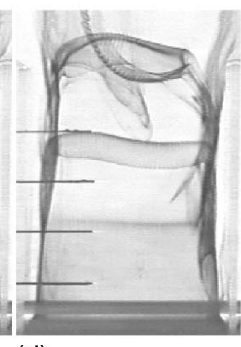

(d)

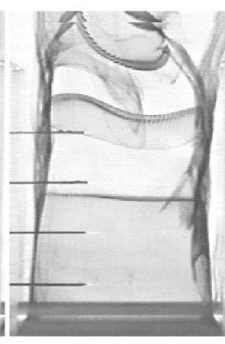

(e)

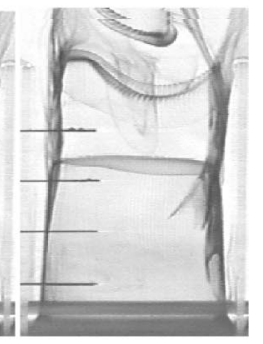

(f)

FIG. 17. Spanwise flow visualization sequence at $\mathrm{AR}=4.0, \mathrm{Re}=300$. (a) $t / T_{\mathrm{v}}=0 ;$ (b) $t / T_{\mathrm{v}}=0.16$; (c) $t / T_{\mathrm{v}}=0.31$; (d) $t / T_{\mathrm{v}}=0.46$; (e) $t / T_{\mathrm{v}}=0.62 ;$ (f) $t / T_{\mathrm{v}}$ $=0.80$.

\section{Transition near $R e=300$}

At $R e=300$, which is within the laminar vortex shedding regime, the flow visualization in Fig. 16(a) shows the roll-up secondary vortices at $x / d \approx 2, z / d=0$, on both sides of the wake. On the right side, the streaklines do not indicate the induction towards and across the cylinder axis, implying no formation of primary vortex. On the left side, however, there is induction and formation of the primary vortex at $x / d \approx 6$. The secondary vortices, after their roll up at $x / d \approx 2$, are engulfed into the rolls of the primary vortices. This figure shows the simultaneous occurrence of the wide wake mode on the right side and the regular shedding mode with the presence of both the primary and secondary vortices on the left side, illustrating the occurrence of these two modes within the laminar vortex shedding regime. ${ }^{9}$

The spanwise visualization of the regular shedding mode at $\mathrm{Re}=300$ indicates two dimensionality of the newly roll-up secondary vortex at $x / d \approx 2$ and the three-dimensional development at $x / d \approx 4$ [Fig. 16(b)]. At the latter position, besides the wavy form, the small-scale vortex seems to be stretched and engulfed into the roll of the developing primary vortex. Further downstream, the secondary vortex becomes three dimensional.

A sequence of the development of the secondary vortices at $\operatorname{Re}=300$ in the regular shedding mode is shown in Figs. 17(a) $-17(\mathrm{f})$. At $t / T_{\mathrm{v}}=0$, the two secondary vortices at $x / d$ $\approx 1.8$ and 3.3 have wavy pattern across the span [Fig. 17(a)]. $T_{\mathrm{v}}$ is the period of the primary vortices. At $t / T_{\mathrm{v}}=0.16$ and 0.31 , the downstream secondary vortex is stretched and engulfed into the primary vortex at $x / d \approx 5$ [Figs. $17(\mathrm{~b})$ and 17(c)]. At $t / T_{\mathrm{v}}=0.46$ and 0.62 , the upstream one seems to experience greater stretching than the proceeding one [Figs. $17(\mathrm{~d})$ and $17(\mathrm{e})]$. It becomes three dimensional at $x / d \approx 6$ at $t / T_{\mathrm{v}}=0.80$ [Fig. 17(f)]. In this sequence, there also seems to be in-phase spanwise development of succeeding vortices, implying feedback mechanism of these vortices.

These two types of vortices have also been shown in the normalized vorticity $\omega_{\mathrm{z}} /\left(U_{\mathrm{o}} / d\right)$ contours (Fig. 13). At the $\mathrm{Re}_{\mathrm{cr}}=320$, the high concentration of vorticity associated with the primary vortex at $x / d \approx 4.5$ extends across the center of the wake. Those associated with the secondary vortices at $x / d \approx 1$ and 1.7 appear on both sides of the wake. At $x / d$ $\approx 2.7$, on both sides of the wake, two secondary vortices undergo "pairing" process. In the present study, pairing in- cludes the merging, pairing into a bigger vortex or collective interaction.

The above flow visualization also indicates that the secondary vortices play an important role in the transition from the laminar wake to wide wake mode. At higher Reynolds numbers, the flow visualization does not show clearly the mutual interaction of the two types of vortices. In order to explore further the effect of the small-scale vortices and their pairings, the Strouhal number $\mathrm{St}_{\theta}$, based on the momentum thickness, of the separated boundary layer in different flow regimes was measured.

\section{E. Transition in higher Reynolds number regimes $\mathbf{3 0 0}$ $<\operatorname{Re}<1.8 \times 10^{3}$}

Using the LDA, the boundary layer momentum thicknesses $\theta$ at $x / d=0$ at the lower Reynolds number range of $260<\operatorname{Re}<1.8 \times 10^{3}$ were obtained (not shown here). They agree with those of other workers. ${ }^{5,10}$ The Strouhal numbers based on the frequencies of the primary vortex $\mathrm{St}_{\theta \mathrm{v}}$ of the secondary vortices $\mathrm{St}_{\theta \mathrm{i}}$, and of the pairing secondary vortices $\mathrm{St}_{\theta i / 2}$ are shown in Fig. 18. For mixing layer, the most amplified wave has a Strouhal number $\mathrm{St}_{\theta}$, based on average velocity of $0.032 .{ }^{37,38}$ For jet instability, the roll-up frequency of exit shear layer is at $\mathrm{St}_{\theta}$ of $0.012^{39,40}$ and is lower than the acoustically excited $\mathrm{St}_{\theta}$ of $0.016 .{ }^{41,42}$ Near the $\mathrm{Re}_{\text {cr }}$ $=320$ of this aspect ratio, the $\mathrm{St}_{\theta \mathrm{i}}$ of the individual secondary vortices is at the most amplified mode of $\mathrm{St}_{\theta}=0.012$. Thus, in the lower transition regime the disturbances of individual secondary vortices themselves excite the Kelvin-Helmholtz instability of the separated shear layer at the most amplified mode. The disturbances associated with primary vortices do not excite the separated shear layer. For the pairing secondary vortices, the $\mathrm{St}_{\theta \mathrm{i} / 2}$ is basically half of the $\mathrm{St}_{\theta \mathrm{i}}$. Based on the ratio of the responses and subharmonic forcing frequencies of mode II ${ }^{34}$ the induced Strouhal number is basically the same as the $\mathrm{St}_{\theta \mathrm{i}}$. Thus, at $\mathrm{Re} \approx 320$, the subharmonic disturbances associated with the pairing secondary vortices also excite the separated shear layer, forming additional individual secondary vortices. The combined excitation of the individual and pairing secondary vortices results in the more rapid increases in the number of secondary vortices (Fig. 5) and in their energy (Figs. 8 and 15). Although pairings of secondary vortices do not play a significant role in the mutual interference with the primary vortex sheet and the for- 


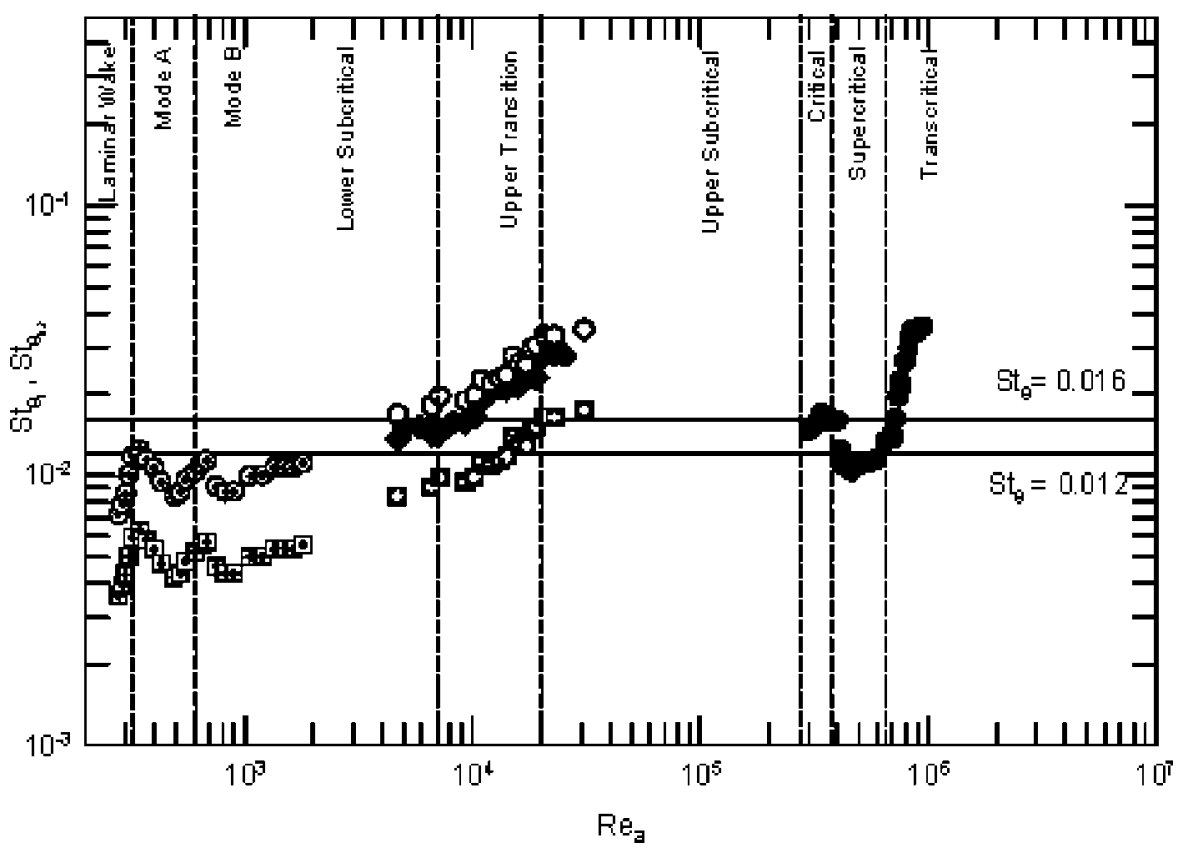

FIG. 18. Strouhal numbers of secondary vortices and paired secondary vortices. $\oplus$, Secondary vortices; $\boxplus$, paired vortices; , secondary vortices, Lo and $\mathrm{Ko}$ (2001) (Ref. 8); $\boldsymbol{H}$, paired vortices, Lo and Ko (2001) (Ref. 8); secondary vortices: grooved cylinder; O, Ho and Huang (1982) (Ref. 34): subharmonic forcing at mode II. mation of primary vortices, nevertheless, their disturbances excite the separated shear layer, resulting in the additional formation and higher energy of the secondary vortices. At $\operatorname{Re}>320$, the increase in the number of secondary vortices redistributes the energy, resulting in the decrease in $\left\langle u_{\mathrm{i}}^{2}\right\rangle / u_{\mathrm{i}}^{2}$ (Fig. 8).

At $\mathrm{Re} \approx 650$, in the transition regime of mode $A$ and mode $B$ subregimes, similar phenomenon of the $\mathrm{St}_{\theta \mathrm{i}}$ and of the mode II of $\mathrm{St}_{\theta \mathrm{i} / 2}$ being at the most preferred mode, is also found (Fig. 18). The more rapid increases in the low-energy secondary vortices, lower of the high-energy vortices, also illustrate the excitation of the separated shear layer due to the individual and pairing secondary vortices (Fig. 5). As the energies of the individual and pairing vortices are higher than those at $\mathrm{Re} \approx 320$ (Fig. 15), one would expect the effect of excitation would be higher. As the number and energy of secondary vortices increase and couple with their upstream shift under the strain of primary vortices, these more closely spaced vortices, under the Biot-Savart induction, interact more mutually with each other, resulting in their threedimensional distortion.

At $\mathrm{Re} \approx 1.8 \times 10^{3}$, the $\mathrm{St}_{\theta \mathrm{i}}$ and the mode II of $\mathrm{St}_{\theta \mathrm{i} / 2}$ are also about 0.012 (Fig. 18). In this transition regime, from the mode $B$ subregime to the lower subcritical regime, similar phenomenon of the excitation at the most preferred mode of the separated shear layer by the individual and pairing secondary vortices also occurs.

\section{F. Transition in upper Reynolds number regimes}

The present investigation concerns the mutual interference of the small-scale and large-scale vortices within the low Reynolds number regimes. The results suggest the transition mechanism that because of the presence of the smallscale secondary vortices, the vortex sheet and the formation of the large-scale primary vortices are affected. In turn, the strain field of the large-scale vortex sheet affects the devel- opment and formation of small-scale vortices. Further, the disturbances of these two types of vortices and the pairings of the small-scale secondary vortices at and near the most preferred mode of excitation also play important role.

The mechanism, with different role of the pairing secondary vortices, was first suggested in the study along with the establishment of the upper transition regime between the lower and upper subcritical regime. ${ }^{8}$ The results of the present investigation in the upper transition regime agree with those of earlier study. Thus, both studies, within the Reynolds number regime of $260<\operatorname{Re}<4 \times 10^{4}$, indicate the significant role of the small-scale secondary vortices, shed from the separated shear layer. It would, then, be desirable to explore whether the same mechanism is responsible for the transition in high flow regimes of the wake. Before the studies of the present authors, ${ }^{14,43}$ the study on the identification and the role of the small-scale vortices in high flow regimes was very scarce. The available results are the earlier results of the present authors on the very near wake of grooved cylinder. The presence of the small-scale secondary vortices within the supercritical and transcritical regimes is established. The reason for the adoption of grooved cylinder in the studies of these high Reynolds number regimes was due to the lack of such facility, even though the unknown effect of the grooves on the wake was recognized. The aspect ratios of the two earlier studies were 4.3 and 5.3, while the study within the upper transition regime of smooth cylinder was $4 .{ }^{8}$ Thus, the aspect ratios are comparable in that the present concept of two dimensionality of the small-scale instability layer was maintained.

Based on the available results, ${ }^{8,14,43}$ the following sections will try to extend the concept of the basic mechanism to other regimes. As the aspect ratios of the studies were about 4 , the division of different flow regimes is based on the following. In the laminar shedding and in the subcritical regimes, the Reynolds number is not adjusted and the $\mathrm{Re}_{\mathrm{cr}}$ 


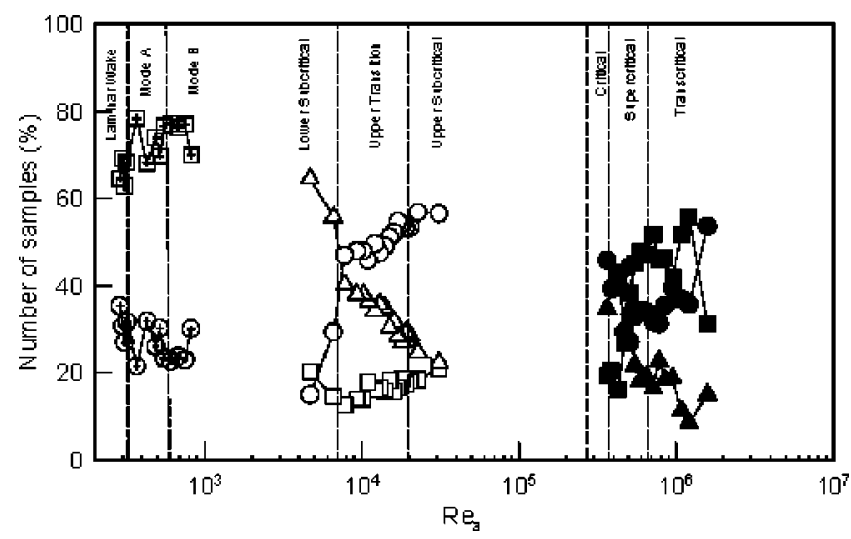

FIG. 19. Numbers of samples of secondary vortices with Reynolds number. $\oplus, x / d=1.2,2<\sigma_{\mathrm{i}}<10$; $\boxplus, x / d=1.2,1<\sigma_{\mathrm{i}}<2$; Lo and Ko (2001) (Ref. 8): $\bigcirc, \sigma_{\mathrm{i}}>3 ; \square, 2<\sigma_{\mathrm{i}}<3 ; \triangle, 1<\sigma_{\mathrm{i}}<2$. Grooved cylinder: $\boldsymbol{\bullet}, \sigma_{\mathrm{i}}>3 ; \mathbf{\square}$, $2<\sigma_{\mathrm{i}}<3 ; \boldsymbol{\Delta}, 1<\sigma_{\mathrm{i}}<2$.

320. The Reynolds numbers $\operatorname{Re}_{\mathrm{a}}$ of higher flow regimes ${ }^{14,43}$ were arbitrarily adjusted to match the Reynolds number of transition from the subcritical to critical regime.

The variations of the percentages of numbers of recovered secondary vortices are shown in Fig. 19. At $\operatorname{Re}_{a}>10^{3}$, the recovery was based on the three threshold levels. In the following sections, to simplify the presentation, the supercritical and transcritical regimes at $\operatorname{Re}_{\mathrm{a}}>10^{5}$ are arbitrarily referred as the high Reynolds number regime, while the laminar shedding and wake transition regimes at $\operatorname{Re}_{\mathrm{a}}<10^{3}$, as the low Reynolds number regime. The regime at $4 \times 10^{3}$ $<\operatorname{Re}_{\mathrm{a}}<4 \times 10^{4}$ is referred as the upper transition regime. In Fig. 19, in the high and low Reynolds number regimes, the high-energy secondary vortices are significantly lower in number than those of the low-energy vortices. The distributions of the latter in both the low and high regimes indicate the same phenomenon of rapid increase in number before transition. In the upper transition regime, however, the highenergy secondary vortices become dominant during transition.

The variations of the maximum recovered energy of the secondary vortices indicate the dominance of the highenergy vortices (Fig. 20). $\left\langle u_{\mathrm{i}}^{2}\right\rangle_{\max }$ is higher, just before and during the transitions of different regimes, indicating that the high-energy secondary vortices play an important role in the transition of all flow regimes.

The recovered maximum downstream to upstream velocity ratios of the primary vortex sheet, $\left\langle u_{\mathrm{v}}\right\rangle_{+1} /\left\langle u_{\mathrm{v}}\right\rangle_{+2}$, are shown in Fig. 21. Within the formation region of primary vortices, without the effect of secondary vortices, one would expect $\left\langle u_{\mathrm{v}}\right\rangle_{+1} /\left\langle u_{\mathrm{v}}\right\rangle_{+2}>1$. In the low Reynolds number regime, the low-energy secondary vortices $\left(\sigma_{\mathrm{i}}<2\right)$ affect slightly the developing vortex sheet with $\left\langle u_{\mathrm{v}}\right\rangle_{+1} /\left\langle u_{\mathrm{v}}\right\rangle_{+2}$ $\approx 1$. The effect of the high-energy ones $\left(\sigma_{\mathrm{i}}>2\right)$ is more significant, mainly before and during the transition. In the upper transition regime, a similar phenomenon is found, though the effect is generally higher. In the high Reynolds number regime, with $\left.\left\langle u_{\mathrm{v}}\right\rangle_{+1} /\left\langle u_{\mathrm{v}}\right\rangle_{+2}\right\rangle 1$, the low-energy vortices $\left(\sigma_{\mathrm{i}}\right.$ $<2)$ do not have significant effect on the sheet. The highenergy ones $\left(\sigma_{\mathrm{i}}>3\right)$ affect the sheet more significantly with

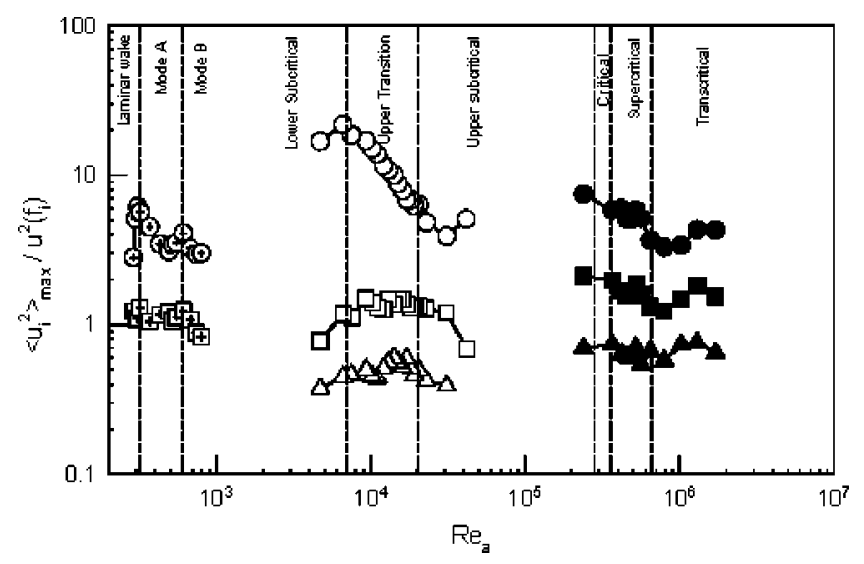

FIG. 20. Maximum recovered energies of secondary vortices with Reynolds number. $\oplus, x / d=1.2,2<\sigma_{\mathrm{i}}<10 ; \boxplus, x / d=1.2,1<\sigma_{\mathrm{i}}<2 ;$ Lo and Ko (2001) (Ref. 8): $\bigcirc, \sigma_{\mathrm{i}}>3 ; \square, 2<\sigma_{\mathrm{i}}<3 ; \triangle, 1<\sigma_{\mathrm{i}}<2$. Grooved cylinder, Lo and Ko (1999) (Ref. 43): $\boldsymbol{O}, \sigma_{\mathrm{i}}>3 ; \boldsymbol{\square}, 2<\sigma_{\mathrm{i}}<3 ; \boldsymbol{\Delta}, 1<\sigma_{\mathrm{i}}<2$.

$\left\langle u_{\mathrm{v}}\right\rangle_{+1} /\left\langle u_{\mathrm{v}}\right\rangle_{+2}<1$. The above results indicate that in different flow regimes the energy of the small-scale secondary vortices is a crucial factor in the development of the largescale vortex sheet.

The maximum linear strain rates $\left[d\left\langle u_{\mathrm{v}}\right\rangle_{3} / d t\right]_{\max } /\left[d\left\langle u_{\mathrm{v}}\right\rangle_{1} / d t\right]_{\max }$ of the most energetic secondary vortices in the three Reynolds number regimes have a significant effect on the maximum recovered energy $\left\langle u_{\mathrm{i}}^{2}\right\rangle_{\max } / u_{\mathrm{i}}^{2}$ of secondary vortices (Fig. 22). Generally, the higher the linear strain rate, the higher is the energy of the secondary vortices. The same trend of the rates in the low and high Reynolds number regimes is found. This agreement might suggest the effect of the grooves in the higher regime is not too significant. In the upper transition regime, however, higher rate is observed, implying that the strain field induces higher energy of small-scaled vortices. This may explain the slightly different phenomenon observed in this regime that there is more rapid increase in the high-energy vortices $\left(\sigma_{\mathrm{i}}>3\right)$. These high-energy ones are the most numerous.

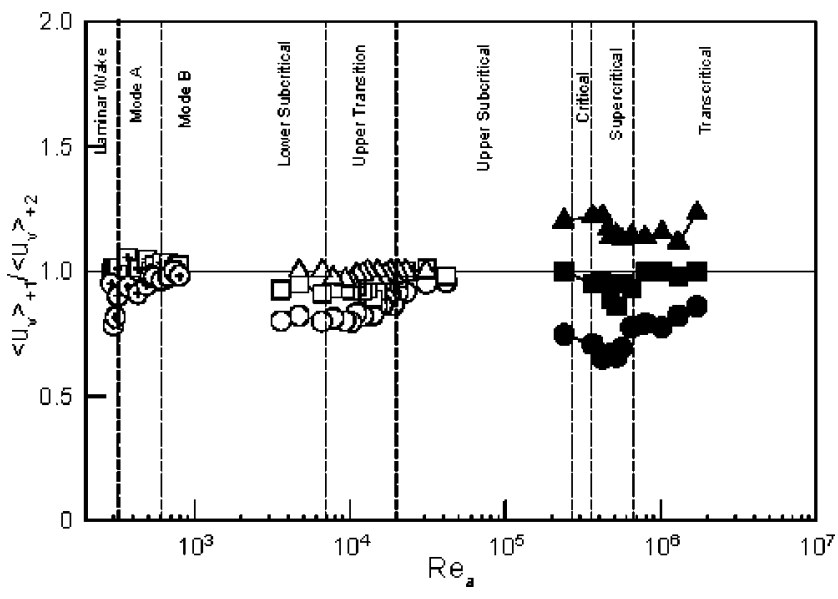

FIG. 21. Recovered velocity ratios with Reynolds number. $\oplus, x / d=1.2,2$ $<\sigma_{\mathrm{i}}<10$; $\boxplus, x / d=1.2,1<\sigma_{\mathrm{i}}<2$; Lo and Ko (2001) (Ref. 8): $\bigcirc, \sigma_{\mathrm{i}}>3 ; \square$, $2<\sigma_{\mathrm{i}}<3 ; \triangle, 1<\sigma_{\mathrm{i}}<2$. Grooved cylinder, Lo and Ko (1999) (Ref. 43): $\sigma_{\mathrm{i}}>3 ; \boldsymbol{\square}, 2<\sigma_{\mathrm{i}}<3 ; \boldsymbol{\Delta}, 1<\sigma_{\mathrm{i}}<2$. 


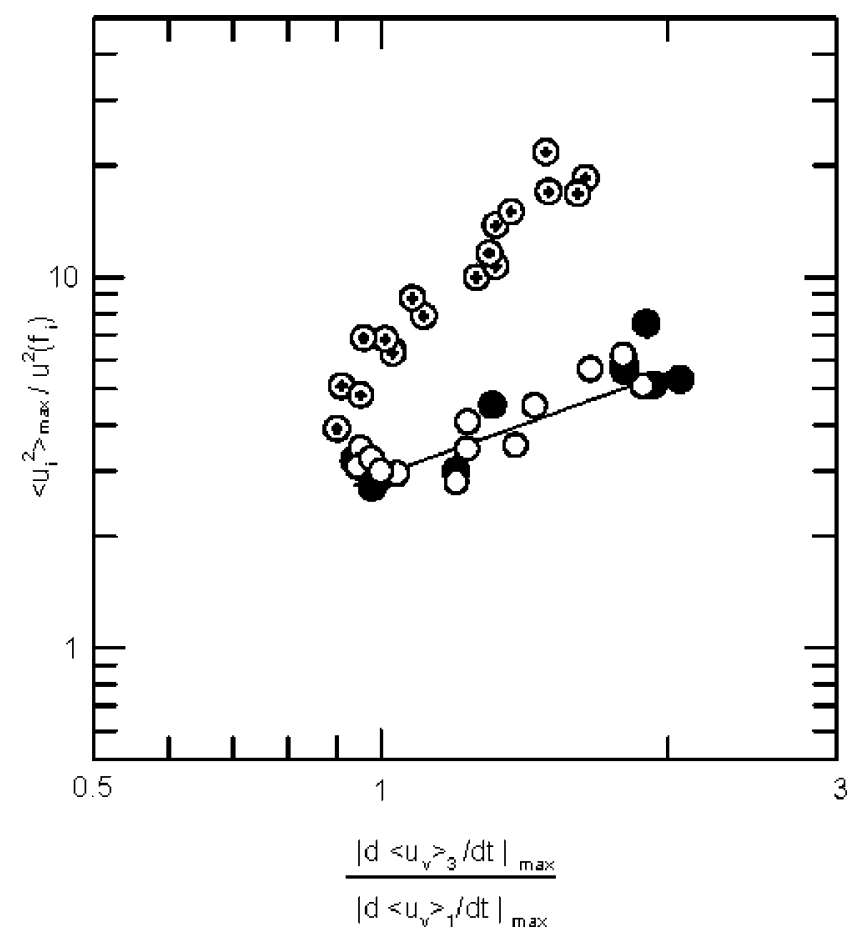

FIG. 22. Maximum strain rate ratios of primary vortex sheet with maximum recovered energies of secondary vortices. $O$, smooth cylinder, $280<\mathrm{Re}_{\mathrm{ad}}$ $<820$; $\oplus$, smooth cylinder, $4 \times 10^{3}<\operatorname{Re}_{a d}<4 \times 10^{4}$; Lo and Ko (2001) (Ref. 8): $\bullet$ grooved cylinder (groove half), $1 \times 10^{4}<\operatorname{Re}_{\mathrm{ad}}<1.2 \times 10^{5}$.

The Strouhal numbers $\mathrm{St}_{\theta}$, based on the momentum thickness near the separation, of the secondary and pairing secondary vortices in the upper transition regime and the high Reynolds number regime are also shown in Fig. 18. Those of the mode II of subharmonic forcing are also shown. In the beginning and during the transitions in different regimes, the $\mathrm{St}_{\theta}$ of the individual and pairing vortices are at and near the most preferred mode of $\mathrm{St}_{\theta}=0.012$ and of the excited one of $\mathrm{St}_{\theta}=0.016$. This agreement over the different flow regimes implies that during transitions the secondary and pairing vortices excite the separated shear layer responsible for the more numerous and more intense small-scale vortices.

\section{CONCLUSIONS}

Based on flow visualization, LDA, DPIV, and a new conditional sampling technique, the present experimental study was carried out in the formation region of the largescale primary vortices within the Reynolds number range of $260<\operatorname{Re}<1.8 \times 10^{3}$. At an aspect ratio of 4 , the range covers the laminar shedding regime, the mode $A$ and the mode $B$ subregimes, and the lower subcritical regime. Based on the conditional sampling technique, the mutual interference of the small-scale secondary vortices and their pairings with the large-scale primary vortex sheet was investigated in details.

Although the low-energy secondary vortices are more numerous, during transition their effect on the developing vortex sheet is not significant. Though their effect is not sig- nificant, their numbers experience rapid increase just before the transition from the laminar shedding regime to the mode $A$ subregime at $\mathrm{Re} \approx 320$ and before and during the transition at $\mathrm{Re} \approx 600$.

The high-energy individual secondary vortices affect the large-scale primary vortex sheet, amplifying the upstream part of the sheet. There is upstream shift of the phase of the recovered peak secondary vortex energy with the trough of the recovered streamwise velocity of the primary vortex sheet. The strain field of the amplified vortex sheet, in turn, enhances the development of small-scale vortices, resulting in the increase in energy and upstream phase shift of secondary vortices. These further enhance the interaction between individual secondary vortices. The low-energy secondary vortices, however, do not affect the vortex sheet. In the formation region of the primary vortices, pairings of secondary vortices are also established. These pairings, be they of highand low-energy ones, do not significantly affect the primary vortex sheet and its roll-up.

Thus, during transitions in the low Reynolds number regime, both the individual secondary vortices and pairing vortices and their increasing numbers excite the separated shear layer at the most preferred mode. The subharmonic forcing of mode II of the increasing number of pairing vortices also plays its role in the increase in number of the individual secondary vortices and their pairings.

As the corresponding result in the supercritical and transcritical regimes of smooth cylinder is not available, the present authors had to rely on those of grooved cylinder. Nevertheless, the findings in these high Reynolds number regimes suggest the same mechanism of transitions.

The present studies indicate the importance of the understanding of small-scale secondary vortices and their pairings within the formation length of large-scale vortices. The understanding of their mutual interference, which is until now neglected by other workers, with the large-scale vortices is also important in further understanding the complicated wake in different flow regimes of cylinder. The existing concept of two dimensionality of flow by other workers, based on large aspect ratio, without considering the two dimensionality of the small-scale Kelvin-Helmholtz instability layer has to be reconsidered. As the Kelvin-Helmholtz instability layer is highly susceptible to disturbances, even within a spanwise cell of small-scale vortices, one would expect the behavior of this layer to be a governing parameter. Any aspect ratios of multiple cells or different from the multiples of a cell span of the small-scale vortices would also involve additional complication of the wake developed. This may be the cause for the significant differences in the results of past workers, who did not pay sufficient attention to this aspect. This may also partly be the cause, until now, for the lack of the basic understanding of the mechanism of transition.

The present study in the regime very near the cylinder was mainly based on conditional sampling technique. Further understanding on the complicated physical phenomenon of downstream development and three-dimensional transition is desirable. 
${ }^{1}$ C. H. K. Williamson, "Vortex dynamics in the cylinder wake," Annu. Rev. Fluid Mech. 28, 477 (1996).

${ }^{2} \mathrm{C}$. H. K. Williamson, "Oblique and parallel modes of vortex shedding in the wake of a circular cylinder at low Reynolds numbers," J. Fluid Mech. 206, 579 (1989).

${ }^{3}$ H. König, M. Elsenlohr, and H. Eckelmann, "The fine structure in the Strouhal-Reynolds number relationship of the laminar wake of a circular cylinder," Phys. Fluids A 2, 1607 (1990).

${ }^{4}$ U. Fey, M. König, and H. Eckelmann, "A new Strouhal-Reynoldsnumber relationship for the circular cylinder in the range $47<\operatorname{Re}<2$ $\times 10^{5}$," Phys. Fluids 10, 1547 (1998)

${ }^{5}$ A. Prasad and C. H. K. Williamson, "The instability of the shear layer separating from a bluff body," J. Fluid Mech. 333, 375 (1997).

${ }^{6} \mathrm{C}$. Y. Wen and C. Y. Lin, "Two-dimensional vortex shedding of a circular cylinder," Phys. Fluids 13, 557 (2001).

${ }^{7} \mathrm{C}$. Norberg, "An experimental investigation of the flow around a circular cylinder: influence of aspect ratio," J. Fluid Mech. 258, 287 (1994).

${ }^{8} \mathrm{~K}$. W. Lo and N. W. M. Ko, "At the upper transition of subcritical regime of a circular cylinder," ASME Trans. J. Fluids Eng. 123, 422 (2001).

${ }^{9}$ C. W. Law and N. W. M. Ko, "Bistable flow in lower transition regime of circular cylinder," Fluid Dyn. Res. 29, 313 (2001).

${ }^{10} \mathrm{M}$. S. Bloor, "The transition to turbulence in the wake of circular cylinder," J. Fluid Mech. 19, 290 (1964).

${ }^{11}$ M. S. Bloor and J. H. Gerrard, "Measurements on turbulent vortices in a cylinder wake," Proc. R. Soc. London, Ser. A 294, 319 (1966).

${ }^{12}$ A. Kourta, H. C. Boisson, P. Chassaing, and H. Ha Minh, "Nonlinear interaction and the transition to turbulence in the wake of a circular cylinder," J. Fluid Mech. 181, 141 (1987)

${ }^{13}$ T. Wei and C. R. Smith, "Secondary vortices in the wake of circular cylinder," J. Fluid Mech. 169, 513 (1986).

${ }^{14}$ K. W. Lo and N. W. M. Ko, "Turbulent near wake behind a partially grooved circular cylinder," ASME Trans. J. Fluids Eng. 119, 19 (1997).

${ }^{15} \mathrm{P}$. K. Stansby, "The effects of end plates on the base pressure coefficient of a circular cylinder," Aeronaut. J. 78, 36 (1974).

${ }^{16}$ S. Szepessy, "On the control of circular cylinder flow by end plates," Euro. J. Mech. B/Fluids 12, 217 (1993).

${ }^{17}$ E. Achenbach, "Distribution of local pressure and skin friction around circular cylinder in cross-flow up to $\operatorname{Re}=5 \times 10^{6}$," J. Fluid Mech. 34, 625 (1968).

${ }^{18}$ O. Guven, C. Farell, and V. C. Patel, "Surface-roughness effects on the mean flow past circular cylinders," J. Fluid Mech. 98, 673 (1980).

${ }^{19}$ J. H. Gerrard, "The wakes of cylindrical bluff bodies at low Reynolds number," Philos. Trans. R. Soc. London, Ser. A 288, 351 (1978).

${ }^{20}$ M. F. Unal and D. Rockwell, "On vortex formation from a cylinder. Part 1: The initial instability," J. Fluid Mech. 190, 491 (1988).

${ }^{21}$ J. H. Gerrard, "The mechanics of the formation region of vortices behind bluff bodies," J. Fluid Mech. 25, 401 (1966).

${ }^{22}$ C. E. Willert and M. Gharib, "Digital particle image velocimetry," Exp. Fluids 10, 181 (1991).
${ }^{23}$ D. L. Reuss, R. J. Adrian, C. C. Landreth, D. T. French, and T. D. Fansler, SAE Technical Series Report No. 890616, 1989.

${ }^{24}$ D. L. Keane and R. J. Adrian, "Theory of cross-correlation of PIV images," Flow Visualization and Images Analysis, edited by F. T. M. Nieuwstadt (Kluwer Academic, Dordrecht, 1993).

${ }^{25}$ A. K. M. F. Hussain, "Coherent structures-reality and myth," Phys. Fluids 26, 2816 (1983).

${ }^{26} \mathrm{C}$. H. K. Williamson, "The existence of two stages in the transition to three-dimensionality of a cylinder wake," Phys. Fluids 31, 3165 (1988).

${ }^{27}$ C. H. K. Williamson, "Three-dimensional aspects and transition," Turbulent Shear Flows 7, edited by F. Durst (Springer, Berlin, 1991).

${ }^{28}$ J. A. Peterka and P. D. Richardson, "Effects of sound on separated flow," J. Fluid Mech. 37, 265 (1969).

${ }^{29}$ S. J. Lin, Ph.D. thesis, University of California, 1981.

${ }^{30}$ J. C. Lasheras, J. S. Cho, and J. Maxworthy, "On the origin and evolution of streamwise vortical structures in a plane free shear layer," J. Fluid Mech. 172, 231 (1986).

${ }^{31}$ T. Cebecci and P. Bradshaw, Momentum Transfer in Boundary Layers (McGraw-Hill, New York, 1977).

${ }^{32} \mathrm{P}$. Freymuth, "On transition in a separated laminar boundary layer," J. Fluid Mech. 25, 683 (1966)

${ }^{33}$ C. M. Ho and N. S. Nosseir, "Dynamics of an impinging jet. Part I. The feedback phenomenon," J. Fluid Mech. 105, 119 (1981).

${ }^{34}$ C. M. Ho and L. S. Huang, "Subharmonics and vortex merging in mixing layers," J. Fluid Mech. 119, 443 (1982).

${ }^{35} \mathrm{C}$. D. Winant and F. K. Browand, "Vortex pairing: the mechanism of turbulent mixing layer growth at moderate Reynolds number," J. Fluid Mech. 63, 237 (1974)

${ }^{36}$ J. C. Lasheras and H. Choi, "Three-dimensional instability of a plane free shear layer: an experimental study of the formation and evolution of streamwise vortices," J. Fluid Mech. 189, 53 (1988).

${ }^{37}$ A. Michalke, "On the inviscid instability of the hyperbolic tangent velocity profile," J. Fluid Mech. 19, 543 (1964).

${ }^{38}$ C. M. Ho and P. Huerre, "Perturbed free shear layer," Annu. Rev. Fluid Mech. 16, 365 (1984)

${ }^{39}$ A. K. M. F. Hussain and M. F. Zedan, "Effects of initial condition on the axisymmetric free shear layer: Effects of the initial-momentum thickness," Phys. Fluids 21, 1475 (1978).

${ }^{40}$ K. B. M. Q. Zaman and A. K. M. F. Hussain, "Vortex pairing in a circular jet under controlled excitation. Part 1. General jet response," J. Fluid Mech. 101, 449 (1980).

${ }^{41}$ K. B. M. Q. Zaman and D. J. McKinzie, "Control of laminar separation over airfoils by acoustic excitation," AIAA J. 29, 1095 (1991).

${ }^{42}$ K. B. M. Q. Zaman, "Effect of acoustic excitation on stalled flows over an airfoil," AIAA J. 30, 1492 (1992).

${ }^{43} \mathrm{~K}$. W. Lo and N. W. M. Ko, "Vortex interaction in the formation region of a grooved circular cylinder," Fluid Dyn. Res. 24, 161 (1999). 